\title{
Mode Shift Keying for Reconfigurable MIMO Antennas: Performance Analysis and Antenna Design
}

\author{
Mehedi Hasan, Israfil Bahceci ${ }^{\circledR}$, Member, IEEE, Md. Asaduzzaman Towfiq ${ }^{\circledR}$, Tolga M. Duman ${ }^{\circledR}$, Fellow, IEEE, \\ and Bedri A. Cetiner ${ }^{\circledR}$, Senior Member, IEEE
}

\begin{abstract}
Space-shift-keying (SSK) and spatial modulation (SM) enable multiple antenna transmission systems to convey information on antenna indices. While SSK/SM helps reduce the number of radio frequency (RF) chains, large numbers of antennas and low spatial correlations are required to achieve high data rates. This work investigates the use and design of multifunctional reconfigurable antennas (MRAs) for SSK/SM based transmission where a single-element MRA generates large numbers of modes. To enhance legacy SSK/SM performance while reducing RF hardware complexity, we propose single- and multi-carrier antenna mode-shift keying (MoSK) and mode modulation (MoM) schemes facilitated by MRAs. Based on an error probability analysis, we determine criteria for MRA design and mode set selection suitable for MoSK/MoM. We also develop two MRA designs and investigate their performances over Rayleigh fading channels. We argue that by creating MRA modes with low pattern correlations, channel correlations can be reduced to improve the detection performance. Extensive simulations demonstrate that MoSK/MoM performance exceeds that of SSK/SM along with significant complexity reduction. For instance, a single-carrier MoSK/MoM using a single MRA with 8 modes achieves about $2 \mathrm{~dB}$ gain compared to legacy SSK/SM requiring 8 antennas, and by multi-carrier MoSK/MoM using 4 subcarriers, an MRA with 32 modes can attain an error rate performance comparable to this single-carrier system.
\end{abstract}

Index Terms-Antenna diversity, MIMO systems, reconfigurable antennas, modulation, multipath channels.

\section{INTRODUCTION}

$\mathbf{S}$ PACE-shift-keying (SSK) [1]-[3] and spatial modulation (SM) [4], [5] are multiple antenna transmission techniques

Manuscript received March 18, 2018; revised August 8, 2018; accepted October 3, 2018. Date of publication October 30, 2018; date of current version January 15, 2019. This work was supported in part by AFOSR under Grant FA 9550-15-1-0040DEF and in part by Defense University Research Instrumentation Program under Grant FA9550-16-1-0352. The review of this paper was coordinated by Prof. T. Kuerner. (Corresponding author: Israfil Bahceci.)

M. Hasan is with the Marvell Semiconductor, Santa Clara, CA 95054 USA (e-mail: mehedi@marvell.com).

I. Bahceci is with the Ericsson Canada, Inc., Kanata, ON K2K 2V6, Canada (e-mail: israfil.bahceci@ericsson.com).

Md. A. Towfiq is with the i5 Technologies, Inc., Logan, UT 84341 USA (e-mail: asaduzzaman.towfiq@i5technologies.com).

T. M. Duman is with the Department of Electrical and Electronics Engineering, Bilkent University, Ankara 06800, Turkey (e-mail: duman@ee. bilkent.edu.tr).

B. A. Cetiner is with the Department of Electrical and Computer Engineering, Utah State University, Logan, UT 84321 USA (e-mail: bedri. cetiner@usu.edu).

Color versions of one or more of the figures in this paper are available online at http://ieeexplore.ieee.org.

Digital Object Identifier 10.1109/TVT.2018.2878768 that rely on modulating the spatial channel to transfer information over a wireless link. In SSK, the information bits determine one of $M$ transmit antenna elements for transmission. With SM, the information bits determine both an antenna index and a constellation signal that is transmitted on the selected antenna. The receiver attempts to decode the information bits by recovering the underlying antenna index (and the constellation signal in case of SM) using the received signal's spatial signature. The main advantage of SSK/SM is the reduction of the number of radio-frequency $(\mathrm{RF})$ chains for multiple antenna transmissions while preserving the diversity gain, i.e., SSK/SM promises simplified hardware with only a small performance trade-off. However, a number of implementation issues exist. For example, in the case of pulse shaping with a period longer than the symbol time, one may need more RF chains to perform SSK modulation [2]. Furthermore, in order to increase the data rate, one needs to increase the number of antennas significantly. This makes SSK/SM suitable for only large units such as base stations or access points. In addition, high spatial correlations among the channels from different transmit elements result in performance degradation [1].

In this paper, we investigate and analyze the SSK and SM for multiple input multiple output (MIMO) systems comprising of multifunctional reconfigurable antenna (MRA) elements. A single MRA element, capable of dynamically changing its properties (radiation pattern, polarization, frequency), a.k.a. modes, provides important degrees of freedom that can be used to significantly enhance the system performance [6]-[11]. Recently, a reconfigurable antenna (RA) based SSK solution has been reported in the literature [12], demonstrating that SSK with polarization reconfigurable antennas performs better than legacy SSK for a line-of-sight multipath propagation model. In [12], the additional degrees of freedom have been obtained by changing the Rician $K$-factor and the polarization correlation coefficient. However, for rich scattering environments where the propagation is typically non-line of sight (NLOS), one needs to utilize additional RA properties to make use of spatial diversity. With this motivation, here we concentrate on both the use and design of MRAs for SSK/SM applications. Towards this goal, we perform a comprehensive analysis of MRAs within this context and present two different MRA designs. Associated with each excited mode of an MRA that is selected for transmission, a different spatial channel is created. Considering a multipath rich scattering propagation medium, we propose to employ radiation pattern reconfigurable antennas for which along with the pattern itself the polarization property over the pattern is reconfigurable, resulting in a large number of modes. 
Each of the modes is designed in such a way that the associated radiation patterns result in low spatial correlations. We name the proposed schemes mode shift keying (MoSK) and mode modulation (MoM).

We develop union bounds on the pairwise error probability (PEP) that relates the underlying radiation patterns, i.e., modes, to the overall error rate performance. We then investigate the MRA design problem and employ the performance analysis results to determine the relevant optimization criteria so as to select the antenna modes suitable for MoSK/MoM based transmissions. We also develop two different MRA designs based on parasitic tuning [13]. Different from earlier MRA designs, this work aims at determining MRA architectures capable of generating a large number of modes resulting in low pattern correlations. We note that the proposed MoSK and MoM schemes establish a similar form of media-based modulation technique proposed in [14] where Khandani investigates the impact of intentional variation of RF properties (permittivity, resistivity, and permeability) of a medium placed nearby the transmitter. In our scheme, the antenna element properties are varied based on the parasitic tuning approach to create different spatial signatures from the same antenna position. Note that this is not possible with legacy SSK schemes as the number of antennas needs to be increased. Since PIN diodes with typical switching times on the order of 10 nanoseconds [15] are employed for the proposed parasitic tuning, very high baud rates can be attained.

Another contribution of this work is the extension of MoSK/MoM schemes to wideband transmissions. Even though SSK and SM have received a considerable attention over the last decade [16]-[19], there are only a limited number of works for wideband transmissions [20]. Legacy SSK/SM proposed for multi-carrier (MC) transmission schemes assume availability of multiple RF chains as opposed to the single-carrier (SC) SSK schemes [21]. However, with a single RF chain, wideband SSK becomes inefficient as one would still need to increase the number of antenna elements to improve the transmission rates [1]. In [20], Sugiura and Hanzo propose an SC based SMMIMO suitable for broadband communications. Although the proposed method attains a near-capacity performance with the aid of a three-stage concatenated architecture, it is still based on SC transmissions. In our work, we propose MC based wideband MoSK and MoM schemes. For the proposed scheme, our analysis on the error probability shows that, thanks to the joint mode and frequency selectivity of the channel, one can increase the number of antenna modes, and hence, the transmission rates over wideband channels considerably.

To summarize, the contributions of this work are threefold: (i) novel design of MRAs optimized for MoSK and MoM based transmissions and performance analysis, (ii) feasible and efficient MoSK and MoM schemes for MC based wideband communications and associated performance analysis, and (iii) extensive numerical examples based on practical MRA designs in realistic settings demonstrating the advantages of the proposed schemes. The proposed design is suitable for a generic propagation environment, i.e., it is not limited to LOS propagation as in [12]. In addition, since the proposed MRA system can generate a large number of modes, in response to the underlying propagation medium characteristics, one can perform a mode subset selection to determine the best set of modes suitable for MoSK and MoM. Since legacy SSK/SM schemes employ MIMO systems that are fixed by design, they do not have such flexibility, and their performance is limited by the underlying propagation medium and the resulting spatial channel statis-

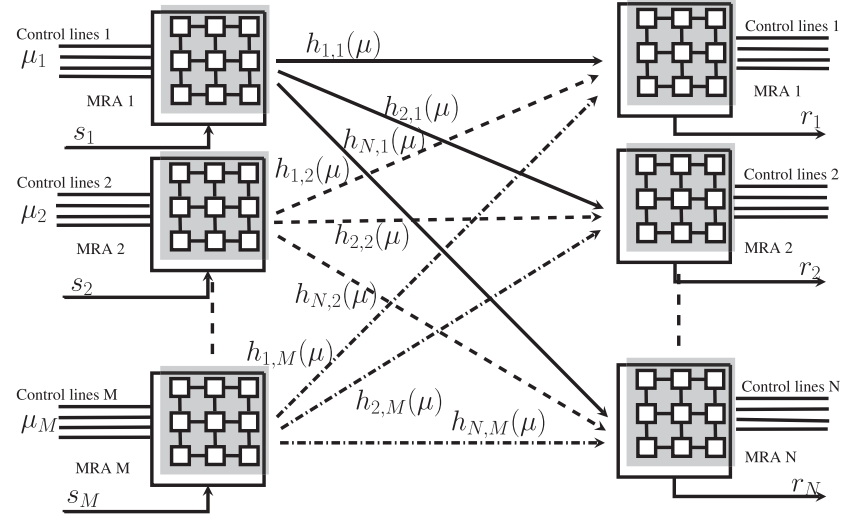

Fig. 1. MIMO channel with $M$ transmit and $N$ receive MRA elements. $\mu_{m}$ : MRA- $m$ mode, $h_{n, m}$ : channel gain from antenna-m to antenna-n, $s_{m}:$ signal from MRA- $m, r_{n}$ : signal received by MRA- $n$. Control lines enable the generation of the modes.

tics. Numerical examples using two different practical MRA designs indicate that the proposed MoSK and MoM schemes for both narrowband and wideband transmission achieve superior performance compared to the legacy SSK/SM schemes. For example, with SC MoSK/MoM, our MRA designs utilizing 8 modes achieve up to $2 \mathrm{~dB}$ SNR gains compared to legacy SSK/SM schemes requiring 8 antenna elements. Furthermore, the proposed MC MoSK/MoM schemes utilizing a 32-mode MRA over 4 subcarriers achieve similar error rates compared to SC MoSK/MoM schemes with 8 modes, that is, the wideband MoSK/MoM design is effective in exploiting both frequency and radiation pattern diversity. Note that an equivalent legacy SSK/SM scheme requires 32 antenna elements to achieve the same data rates, indicating that large reductions in complexity with the MoSK/MoM schemes are possible.

The rest of the paper is organized as follows. In the next section, we describe the system and channel models for MRA systems. In Section III, the newly proposed MoSK and MoM schemes along with analytical calculations of error probabilities are presented. MoSK/MoM for wideband transmission is explained in Section IV. Section V provides details of the practical MRAs designed by full-wave electromagnetic analysis [22]. Simulation results for both narrowband and wideband transmissions are provided in Section VI. Finally, we conclude the paper in Section VII.

\section{Channel And System Model}

\section{A. MIMO Channel With MRAs}

Let us consider a wireless MIMO channel with $M$ transmit and $N$ receive MRA elements, as illustrated in Fig. 1. For brevity, we assume that the receive antenna elements are fixed to some configuration state and are not varied throughout the transmissions. Let $\vec{f}(\theta, \phi, \mu)$ denote the complex far-field radiation pattern where $\mu \in\left\{1,2, \ldots, L_{\mu}\right\}$ represents the antenna mode. For MIMO systems comprising of MRAs (MR-MIMO) with $M$ MRA elements, the complex E-field pattern for the $m$ th element can be expressed as

$$
\vec{f}_{m}(\theta, \phi, \mu)=f_{\theta, m}(\theta, \phi, \mu) \vec{e}_{\theta}+f_{\phi, m}(\theta, \phi, \mu) \vec{e}_{\phi}
$$

where $f_{\theta}$ and $f_{\phi}$ are the $\theta$ and $\phi$ components of the radiated electric field along the directions of $\vec{e}_{\theta}$ and $\vec{e}_{\phi}$ unit vectors, respectively. The variable $\mu \in \mathcal{M}=\left\{1, \ldots, L_{\mu}\right\}$ denotes the 
mode index of antenna- $m, m=1, \ldots, M$. Since each element can have a different configuration resulting in a different radiation pattern, the channel impulse response will be a function of the antenna radiation pattern, i.e., the mode. Assuming that the receiver configuration is fixed, we denote the channel impulse response from MRA- $m$ to MRA- $n$ as $h_{n, m}(\mu)$ where $\mu$ emphasizes the dependency of the channel realization on the transmit MRA mode. Using the beamspace representation in a doubledirectional MIMO channel model [23], [24], one can show that the impact of transmit antenna pattern can be decoupled from all other propagation related terms as follows [25]

$$
h_{n, m}(\mu)=\boldsymbol{\alpha}_{\mu}^{H} \boldsymbol{x}_{n, m}
$$

where $\boldsymbol{\alpha}_{\mu}=\left[\alpha_{\mu, 1} \ldots \alpha_{\mu, F}\right]$ represents the $F \leq L_{\mu}$ synthesis coefficients for the radiation pattern $\vec{f}(\theta, \phi, \mu), \mu=1, \ldots, L_{\mu}$, and $\boldsymbol{x}_{n, m}$ is the $F \times 1$ column vector obtained by projecting the channel gains onto the $F$ basis patterns and represents the effect of antenna orientations, receive antenna pattern, propagation delays, steering vectors, and the path gains. $(\cdot)^{H}$ denotes the conjugate transpose.

We assume that only one of the available antennas is activated during the transmission at any given time. Then, we have the overall channel as an $N \times 1$ vector $\boldsymbol{h}_{m}(\mu)$ with $m$ indicating the transmit antenna index. We define two covariance matrices: (i) the covariance matrix for a fixed mode between the channels from the transmit antennas $m_{1}$ and $m_{2}$ to the receive antennas as

$$
\Psi_{m_{1}, m_{2}}=E\left[\boldsymbol{h}_{m_{1}}(\mu) \boldsymbol{h}_{m_{2}}(\mu)^{H}\right]
$$

and (ii) the covariance matrix from the transmit antenna- $m$ to the receive antennas for different antenna modes, $\mu_{i}$ and $\mu_{j}$ :

$$
\Upsilon_{\mu_{i}, \mu_{j}}=E\left[\boldsymbol{h}_{m}\left(\mu_{i}\right) \boldsymbol{h}_{m}\left(\mu_{j}\right)^{H}\right] .
$$

For narrowband transmission, the input-output relation can be expressed as

$$
\boldsymbol{r}(\mu)=\sqrt{P} \boldsymbol{h}_{m}(\mu) s+\boldsymbol{n}
$$

where $\boldsymbol{r}(\mu)$ is the received signal vector $\boldsymbol{r}(\mu)=\left[r_{1}(\mu) \cdots\right.$ $\left.r_{N}(\mu)\right]^{T}, s$ is the transmitted waveform normalized to unit energy, $P$ is the transmit power, and $\boldsymbol{n}=\left[n_{1} \cdots n_{N}\right]$ denotes the additive white Gaussian noise (AWGN) with the zero-mean circularly symmetric complex Gaussian distribution having a variance of $\sigma^{2} / 2$ per dimension. Assuming the presence of a large number of paths with no LOS components, and using the well known central limit theorem, the channel matrix exhibits a multivariate circularly symmetric complex Gaussian distribution, i.e., in what follows, we assume that the channel undergoes spatially and temporally correlated Rayleigh fading.

\section{B. SSK/SM With Single-Mode MIMO}

Assume a legacy MIMO antenna for which $\mu$ is fixed for each antenna- $m$. For SSK, the transmitted bits determine the active antenna index $m$ from which a waveform signal is transmitted. With SM transmission, additional information is sent using a signal from a constellation $\mathcal{S}=\left\{s_{1}, \ldots, s_{Q}\right\}$ where $Q=2^{r}$ is the number of symbols in the constellation with $r$ denoting the number of bits per symbol. Hence, SM with $Q=1$ and $s_{1}$ denoting the waveform reduces to SSK modulation. The maximum likelihood decoder (MLD) with ideal channel state information to detect the antenna index (and also the symbol in case of SM) is given by [4]

$$
(\hat{m}, \hat{s})=\arg \min _{m \in\{1, \ldots, M\}, s \in \mathcal{S}}\left\|\boldsymbol{r}(\mu)-\sqrt{P} \boldsymbol{h}_{m}(\mu) s\right\|
$$

where $\|\cdot\|$ denotes the Euclidean norm. Assuming that the channel is fixed for a transmitted waveform $s$ normalized to unit energy, Chernoff bound on the pairwise error probability (PEP) of the MLD can be expressed as [26]

$$
\begin{aligned}
P\left(m \rightarrow \hat{m}, s \rightarrow \hat{s} \mid \boldsymbol{h}_{m}(\mu), s\right) \leq \\
\quad \exp \left(-\frac{P}{4 \sigma^{2}}\left\|s \boldsymbol{h}_{m}(\mu)-\hat{s} \boldsymbol{h}_{\hat{m}}(\mu)\right\|_{F}^{2}\right) .
\end{aligned}
$$

With $Q=1$, we obtain the SSK modulation for which the bound on PEP averaged over the Rayleigh fading channel statistics can be obtained from (7) as [27]

$$
P(m \rightarrow \hat{m}) \leq\left|I_{N}+\frac{P}{4 \sigma^{2}} \Psi_{m / \hat{m}}\right|^{-1}
$$

where

$$
\Psi_{m / \hat{m}}=\Psi_{m, m}+\Psi_{\hat{m}, \hat{m}}-\Psi_{m, \hat{m}}-\Psi_{\hat{m}, m}
$$

is the covariance matrix of the channel difference between antenna $m$ and $\hat{m}$.

\section{Mode ShIFt Keying And Mode Modulation}

\section{A. Constellation}

The legacy SSK/SM schemes for a MIMO system and the proposed MoSK and MoM schemes for a single MRA system are shown in Figs. 2(a) and 2(b), respectively. The SSK/SM scheme for which the antenna configuration is fixed and independent from the transmitted information [2], [4], [5] requires multiple antenna elements and a single-pole multi-throw RF switch to perform antenna selection, whereas the proposed MoSK/MoM scheme requires only a single MRA element where the information bits determine the antenna mode for each channel use. The baseband part of both MoSK/MoM and legacy SSK/SM scheme are similar to each other. The SSK/SM (MoSK/MoM) Operation Enabler block decides on the underlying (i) SSK/SM (MoSK/MoM) method and (ii) waveform generator $(\mathrm{SSK} / \mathrm{MoSK}) /$ modulator $(\mathrm{SM} / \mathrm{MoM})$, based on the channel state information feedback and/or some system parameters. For MoSK/MoM, by selecting $L_{a}$ modes out of the available $L_{\mu}$, the transmitter can map $R=\log _{2}\left(L_{a}\right)$ bits as the index of the selected modes. For ease of exposition, here, we assume that the $L_{a}$ is a power of two, i.e., $L_{a}=2^{R}$, where $R$ is an integer denoting the number of bits transmitted per MRA mode. In Table I, we provide examples of MoSK and MoM mapping with four selected modes. In the case of MoM, for this example, a BPSK symbol is carried on top of MoSK.

The maximum data rate for SSK/SM and MoSK/MoM can be expressed as $\frac{\log _{2}(X)+\log _{2}(Q)}{T_{s w}}$ where $X=M$ for SSK and $X=L_{a}$ for MoSK, and $Q=2^{r}$ is the constellation size (with $r=0$ for SSK and $r \geq 1$ for SM/MoM) [28]. $T_{s w}$ is the switching time for the single-pole multi-throw (SPMT) switches for the SSK/SM and PIN diode control switches for MoSK/MoM. The SPMT switching time can range from 20 nanoseconds to 300 micro seconds [28], [29] and it increases as the number of antennas increase. The PIN diode switching time for MRAs can be as low as 4 to 10 nanoseconds [30] depending on reverse bias levels of the PIN driver circuitry. Hence, one would expect 5 times or more throughput with MoSK/MoM when $M=L_{a}$. 


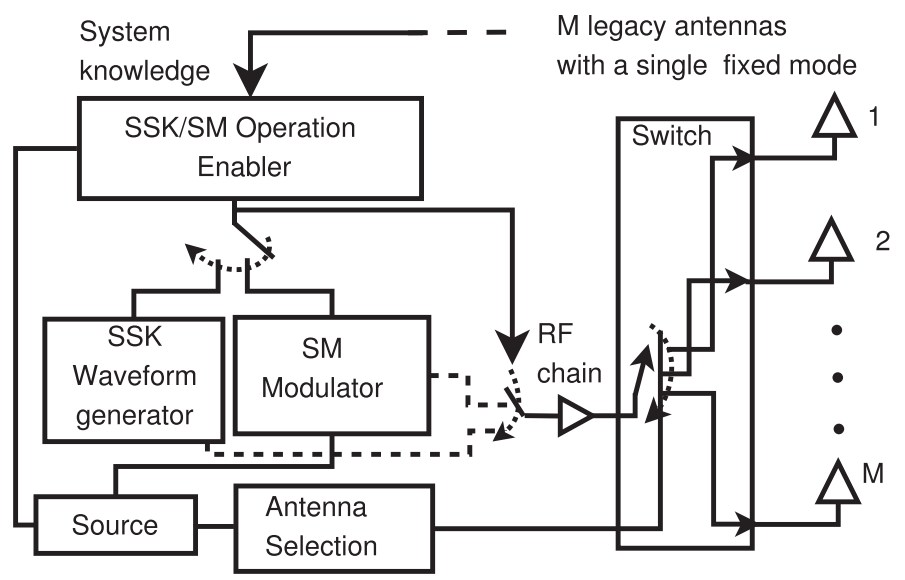

(a)

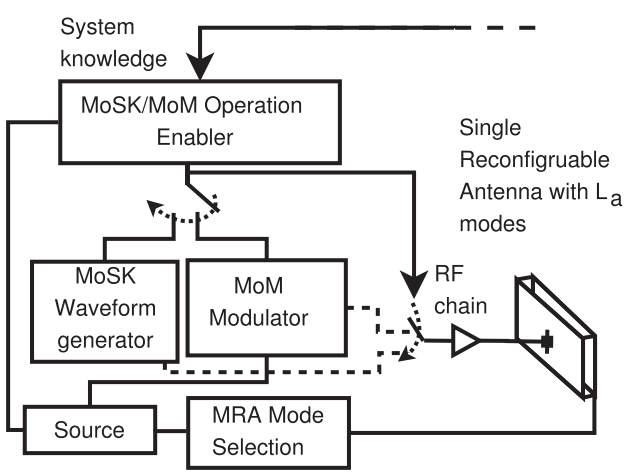

(b)

Fig. 2. Comparison of (a) legacy SSK/SM scheme with $M$ antenna elements where each antenna has one fixed mode, and (b) proposed MoSK/MoM scheme using a single MRA capable of generating $L_{a} \geq M$ modes.

TABLE I

AN EXAMPLE OF MOSK AND MOM MAPPING

\begin{tabular}{|c|c|c|c|}
\hline \multirow[t]{2}{*}{ Selected Mode } & \multirow{2}{*}{$\begin{array}{c}\text { MoSK } \\
(2 \text { bits/symbol/channel }) \\
\text { Input Bits }\end{array}$} & \multicolumn{2}{|c|}{$\begin{array}{c}\text { MoM } \\
(3 \mathrm{bits} / \mathrm{symbol} / \mathrm{channel})\end{array}$} \\
\hline & & Input Bits & BPSK symbol \\
\hline \multirow{2}{*}{1} & \multirow{2}{*}{00} & 000 & -1 \\
\hline & & 001 & 1 \\
\hline \multirow{2}{*}{2} & \multirow{2}{*}{01} & 010 & -1 \\
\hline & & 011 & 1 \\
\hline 3 & 10 & 100 & -1 \\
\hline \multirow[b]{2}{*}{4} & \multirow[b]{2}{*}{11} & $\frac{101}{110}$ & $\frac{1}{-1}$ \\
\hline & & 111 & 1 \\
\hline
\end{tabular}

For a general spatial modulation scheme with $M$ antennas, $L_{\mu}$ modes per antenna, and a signal constellation with $Q$ elements, the transmitted bits select the antenna index, antenna mode index and constellation signal. The MLD for this scheme can be expressed as

$$
(\hat{m}, \hat{\mu}, \hat{s})=\underset{m \in\{1, \ldots, M\}, \mu \in\left\{1, \ldots, L_{\mu}\right\}, s \in \mathcal{S}}{\arg \min }\left\|\boldsymbol{r}(\mu)-\sqrt{P} \boldsymbol{h}_{m}(\mu) s\right\| .
$$

Note that the transmission according to (5) with fixed $\mu$ along with the MLD in (10) reduces to the legacy SSK schemes employing non-reconfigurable antennas as in (6). Furthermore, the case with $M=1$ and $Q=1$ corresponds to MoSK while the case with $M=1, Q>1$ corresponds to the MoM proposed above.

\section{B. Performance Gains With MoSK/MoM}

From (8) and (9), it is seen that the performance of a legacy SSK system is limited by the original MIMO design, e.g., fixed interelement spacing and array geometry. The proposed MoSK and MoM systems create a flexible single-element antenna platform whose modes can be adapted to the multipath propagation medium for improved performance. To that end, for $M=L_{a}$, i.e., for SSK and MoSK schemes with identical rates, let us define the relative gain between the SSK and MoSK as

$$
\zeta=\frac{\sum_{\hat{m} \neq m} d(m, \hat{m})^{-1}}{\sum_{\hat{\mu} \neq \mu} d(\mu, \hat{\mu})^{-1}}
$$

where $d(m, \hat{m})^{-1}=\left|\Psi_{m / \hat{m}}\right|$ and $d(\mu, \hat{\mu})^{-1}=\left|\Upsilon_{\mu / \hat{\mu}}\right|$ with

$$
\Upsilon_{\mu / \hat{\mu}}=\Upsilon_{\mu, \mu}+\Upsilon_{\hat{\mu}, \hat{\mu}}-\Upsilon_{\mu, \hat{\mu}}-\Upsilon_{\hat{\mu}, \mu} .
$$

A large $\zeta$ indicates that the performance loss of MoSK due to correlations among different antenna modes will be lower compared to that of SSK due to spatial channel correlations among the channels from different antenna elements. This metric is based on an upper bound on the symbol error rates as developed in the Appendix.

\section{Antenna Mode Set Construction for MoSK/MoM}

With an RA system, the loss in the channel gains due to the correlations can be reduced by selecting the RA modes for which $d(\mu, \hat{\mu})^{-1}$ is minimized. This provides additional degrees of freedom for optimized designs compared to the legacy SSK schemes where the MIMO structure is fixed. Assuming that the covariance matrices for all the modes are available, using (11), we obtain the following mode design rule

$$
\begin{gathered}
\mathcal{M}_{a}^{*}=\underset{\mathcal{M}_{a} \subseteq \mathcal{M}}{\arg \min } \sum_{\hat{\mu} \neq \mu,(\mu, \hat{\mu}) \in \mathcal{M}_{a}} d(\mu, \hat{\mu})^{-1} \\
\text { s.t. } \mathcal{M}=\left\{\mu: \operatorname{tr}\left(\Upsilon_{\mu, \mu}\right) \geq \zeta_{t h}\right\}
\end{gathered}
$$

where the search space of the modes are constrained to those resulting in an average SNR of at least $P \zeta_{t h} / \sigma^{2}$ with $\zeta_{t h}$ denoting a threshold parameter. While this criterion aims to determine the set of modes minimizing the average decoding error, the search is computationally complex if $L_{\mu}$ is large. Observing that larger values of $d(\mu, \hat{\mu})$ result in smaller error probabilities, we propose an iterative (greedy) mode design approach as follows:

$$
\begin{aligned}
& \mu_{l}^{*}=\underset{\mu \in \mathcal{M}, \mu \neq \mu_{k}, k=1, \ldots, l-1}{\arg \max }\left(\min _{i=1, \ldots, l-1} d\left(\mu_{i}, \mu\right)\right), \\
& \text { s.t. } \mu_{1}^{*}=\underset{\mu \in \mathcal{M}}{\arg \max } \operatorname{tr}\left(\Upsilon_{\mu, \mu}\right),
\end{aligned}
$$

where $\mu_{l}$ denotes the mode selected at iteration- $l, l=1, \ldots, L_{a}$, the minimization inside the parenthesis ensures that the newly selected antenna mode at iteration- $l$ maximizes the smallest possible $d\left(\mu_{i}, \mu\right)$, and finally the trace condition sets the initial mode to the one achieving the highest SNR among all available modes.

Note that the above design approach assumes that the channel covariance matrices are known. The impact of MRA element radiation pattern on the channel difference covariance matrix 
can be explicitly obtained by substituting the channel model in (2) to (12) and after some manipulation:

$$
\Upsilon_{\Delta}=\left(I_{N} \otimes\left(\boldsymbol{\alpha}_{\mu}^{H}-\boldsymbol{\alpha}_{\hat{\mu}}^{H}\right)\right) \overline{\boldsymbol{R}}\left(I_{N} \otimes\left(\boldsymbol{\alpha}_{\mu}-\boldsymbol{\alpha}_{\hat{\mu}}\right)\right)
$$

where $[\overline{\boldsymbol{R}}]_{\left(n_{1}-1\right) N+f_{1},\left(n_{2}-1\right) N+f_{2}}=\left[\boldsymbol{R}_{n_{1}, n_{2}}\right]_{f_{1}, f_{2}}, f_{1}, f_{2} \in\{1$, $\ldots, F\}, n_{1}, n_{2} \in\{1, \ldots, N\}$ with $\boldsymbol{R}_{n_{1}, n_{2}}=E\left[\boldsymbol{x}_{n_{1}, m} \boldsymbol{x}_{n_{2}, m}^{H}\right]$. Under the rich scattering assumption, the extended covariance matrix $\overline{\boldsymbol{R}}$ becomes positive-definite. Using (15) in the Chernoff bound, we arrive at

$$
\bar{P}(\mu \rightarrow \hat{\mu}) \leq\left|I_{N F}+\frac{P}{4 \sigma^{2}}\left(I_{N} \otimes \boldsymbol{\Xi}_{\mu, \hat{\mu}}\right) \overline{\boldsymbol{R}}\right|^{-1}
$$

where $\boldsymbol{\Xi}_{\mu, \hat{\mu}}=\left(\boldsymbol{\alpha}_{\mu}-\boldsymbol{\alpha}_{\hat{\mu}}\right)\left(\boldsymbol{\alpha}_{\mu}^{H}-\boldsymbol{\alpha}_{\hat{\mu}}^{H}\right)$ is the pattern difference product matrix. Observing that $I_{N} \otimes \boldsymbol{\Xi}_{\mu, \hat{\mu}}$ has $N$ nonzero identical eigenvalues $\left\|\boldsymbol{\alpha}_{\mu}-\boldsymbol{\alpha}_{\hat{\mu}}\right\|^{2}, \overline{\boldsymbol{R}}$ is positive definite, and the product $\boldsymbol{B}(\mu, \hat{\mu})=\left(I_{N} \otimes \boldsymbol{\Xi}_{\mu, \hat{\mu}}\right) \overline{\boldsymbol{R}}$ has $N$ nonzero eigenvalues, using Theorems 1 and 2 in [31], we can write

$$
\begin{gathered}
\left\|\boldsymbol{\alpha}_{\mu}-\boldsymbol{\alpha}_{\hat{\mu}}\right\|^{2 N} \prod_{n=1}^{N} \lambda_{N F-n+1}(\overline{\boldsymbol{R}}) \leq \prod_{n=1}^{N} \lambda_{n}(\boldsymbol{B}(\mu, \hat{\mu}))(\overline{\boldsymbol{R}}) \\
\leq\left\|\boldsymbol{\alpha}_{\mu}-\boldsymbol{\alpha}_{\hat{\mu}}\right\|^{2 N} \prod_{n=1}^{N} \lambda_{n}(\overline{\boldsymbol{R}})
\end{gathered}
$$

where $\lambda_{i}(\boldsymbol{A})$ denotes the $i$ th largest eigenvalue of the Hermitian matrix $\boldsymbol{A}$. Hence, we can write a lower bound on $d(\mu, \hat{\mu})$ as

$$
d(\mu, \hat{\mu}) \geq\left\|\boldsymbol{\alpha}_{\mu}-\boldsymbol{\alpha}_{\hat{\mu}}\right\|^{2 N} \prod_{n=1}^{N} \lambda_{N F-n+1}(\overline{\boldsymbol{R}}) .
$$

Notice that the bounds decouple the impact of antenna pattern from that of $\overline{\boldsymbol{R}}$ allowing us to perform mode optimization based only on the radiation patterns. Thus, a suboptimal mode design based on the optimization in (13) can be expressed as

$$
\begin{aligned}
& \mathcal{M}_{a}^{*}=\underset{\mathcal{M}_{a} \subseteq \mathcal{M}}{\arg \min } \sum_{\hat{\mu} \neq \mu,(\mu, \hat{\mu}) \in \mathcal{M}_{a}}\left\|\boldsymbol{\alpha}_{\mu}-\boldsymbol{\alpha}_{\hat{\mu}}\right\|^{-2 N} \\
& \text { s.t. } \mathcal{M}:\left\{\mu: \iint_{\mathcal{R}}\|\vec{f}(\theta, \phi, \mu)\|^{2} \sin (\theta) d \theta d \phi \geq \gamma_{t h}\right\}
\end{aligned}
$$

where the set of modes in the search space $\mathcal{M}$ is constrained to those having the most of the radiated power within the region $\{(\theta, \phi) \in \mathcal{R}\}$. This region may be obtained from the knowledge of the azimuth and elevation angle spreads of the underlying propagation environment.

Observing that $\max _{\mu, \hat{\mu}} d_{l}(\mu, \hat{\mu})=\min _{\mu, \hat{\mu}} \Re\left\{\boldsymbol{\alpha}_{\mu}^{H} \boldsymbol{\alpha}_{\hat{\mu}}\right\}$, the iterative design of (14) can be simplified to

$$
\begin{aligned}
& \mu_{l}^{*}=\underset{\mu \in \mathcal{M}, \mu \neq \mu_{k}, k=1, \ldots, l-1}{\arg \min }\left(\max _{i=1, \ldots, l-1} \Re\left\{\boldsymbol{\alpha}_{\mu}^{H} \boldsymbol{\alpha}_{\hat{\mu}}\right\}\right), \\
& \text { s.t. } \mu_{1}=\underset{\mu \in \mathcal{M}}{\arg \max }\left\|\vec{f}\left(\theta_{0}, \phi_{0}, \mu\right)\right\|,
\end{aligned}
$$

for $l=1, \ldots, L_{a}$, where the search space $\mathcal{M}$ is again selected according to (20). For the initial mode, $\left(\theta_{0}, \phi_{0}\right)$ can be chosen from a set of direction of interest for antenna coverage. This design ensures that the active antenna modes include those having the least correlations amongst each other while their radiation patterns cover the angular spread region of interest.

The Chernoff bound in (16) along with the product bounds in (17) also clarify that the error performance is dominated by the antenna mode pairs having the smallest Euclidean distance between the corresponding radiation patterns. Assuming identical total radiated power for each antenna mode, i.e., $\left\|\boldsymbol{\alpha}_{\mu}\right\|^{2}=P_{0}$, for $\mu=1, \ldots, L_{\mu}$, we can express the smallest Euclidean distance criteria in terms of the normalized pattern correlation

$$
\rho_{i, j}=\frac{\left|\boldsymbol{\alpha}_{\mu_{i}}^{H} \boldsymbol{\alpha}_{\mu_{j}}\right|}{\left\|\boldsymbol{\alpha}_{\mu_{i}}\right\|\left\|\boldsymbol{\alpha}_{\mu_{j}}\right\|}
$$

between the antenna modes $\mu_{i}$ and $\mu_{j}$. This observation can be employed to optimize the radiation patterns of $L_{\mu}$ modes resulting from the MRA design for MoSK/MoM, where the modes of the MRA shall have as small pattern correlations as possible to maximize the smallest distance between the underlying radiation patterns.

\section{MOSK/MOM FOR WIDEBAND TRANSMISSION}

$5 \mathrm{G}$ and beyond systems heavily rely on MIMO and wideband communications in the form of multicarrier (MC) transmissions (e.g., MIMO-OFDM) [32], [33]. Spatial modulation is therefore critical for various $5 \mathrm{G}$ applications, especially when the design costs for MIMO systems become formidable. The MoSK/MoM schemes create cost-effective alternatives for MC systems where the RAs can create vector channel states (over frequency and antenna modes) and can improve the detection of the underlying mode with almost no hardware complexity increase. To analyze the MoSK/MoM performance for this case, let us assume a wideband transmission with $K$ subcarriers. The signal model in (5) can be extended to the MC case by stacking the $K N \times 1$ column vectors into matrices as

$$
\boldsymbol{R}(\mu)=\sqrt{P} \boldsymbol{H}(\mu) \boldsymbol{S}+\boldsymbol{N}
$$

where $\boldsymbol{R}(\mu)=\left[\boldsymbol{r}_{1}(\mu) \cdots \boldsymbol{r}_{K}(\mu)\right]$ is the $N \times K$ received signal matrix, $\boldsymbol{H}=\left[\boldsymbol{h}_{1}(\mu) \cdots \boldsymbol{h}_{K}(\mu)\right]$ is the $N \times K$ channel matrix for MRA mode- $\mu, \boldsymbol{S}=\operatorname{diag}(s, \ldots, s)$ and $\boldsymbol{N}=\left[\boldsymbol{n}_{1} \cdots \boldsymbol{n}_{K}\right]$ is AWGN matrix. The Chernoff bound on the PEP for the MLD in this case can be obtained as

$$
\begin{aligned}
P(\mu & \rightarrow \hat{\mu} \mid \boldsymbol{H}(\mu), \boldsymbol{H}(\hat{\mu})) \\
& \leq \exp \left(-\frac{P}{4 \sigma^{2}} \sum_{k=1}^{K}\left\|\boldsymbol{h}_{k}(\mu)-\boldsymbol{h}_{k}(\hat{\mu})\right\|_{F}^{2}\right)
\end{aligned}
$$

Let the subcarrier spacing be $\Delta f \mathrm{~Hz}$, and assume that the channel has a coherence bandwidth of $W_{c}=\digamma \Delta f$, with $\digamma$ denoting the number of subcarriers within the coherence bandwidth, while the overall transmission bandwidth, (denoted by) $W_{B}$, is much higher than the coherence bandwidth, i.e., $W_{c} \ll W_{B}$. We assume that the channel is block fading such that the total transmission band consists of $K$ independently fading coherence blocks, i.e., $W_{B}=K W_{c}$.

We consider two different transmission scenarios: (i) subband transmission with frequency interleaving, and (ii) wholeband transmission. For the former case, assuming a subcarrier shuffling using a uniform interleaver, the subcarriers assigned to the recipient become almost independent as long as the number of subcarriers in the subband is at most $K$. Hence, assuming that such an interleaving is possible, the joint probability density function can be expressed as the product of individual probability density functions, and the PEP upper bound averaged over 
the channel statistics can be obtained as,

$$
P_{S B}(\mu \rightarrow \hat{\mu}) \leq \prod_{k=1}^{K}\left|I_{N}+\frac{P}{4 \sigma^{2}} \Upsilon_{\mu / \hat{\mu}, k}\right|^{-1}
$$

where $\Upsilon_{\mu / \hat{\mu}, k}$ is the covariance matrix of the channel difference vector at the subcarrier $k$. For the whole-band transmission, a similar PEP analysis results in

$$
P_{W B}(\mu \rightarrow \hat{\mu}) \leq \prod_{k=1}^{K}\left|I_{N}+\frac{P \digamma}{4 \sigma^{2}} \Upsilon_{\mu / \hat{\mu}, k}\right|^{-1}
$$

where $\Upsilon_{\mu / \hat{\mu}, k}$ in this case refers to the covariance matrix of the channel for the $k$ th coherence block. It is seen that decoding the antenna mode using a larger number of channels that are independent from each other, the PEP will become smaller, which is expected due to the availability of the additional frequency diversity. This implies that the number of modes to convey information can be increased to achieve the same PEP, and thus, the data rates can be increased. It is also seen that if the frequency selectivity of the channel is higher, i.e., for smaller $\digamma$, a larger number of modes can be employed for MoSK/MoM. Note that with legacy SSK schemes using legacy antennas with fixed properties, wideband transmission is very inefficient and may be impractical as one has to increase the number of antenna elements to increase the data rate.

The PEP bound for MoM using MC transmission can similarly be obtained as

$$
P(\mu \rightarrow \hat{\mu}, \boldsymbol{S} \rightarrow \hat{\boldsymbol{S}}) \leq \prod_{k=1}^{K}\left|I_{N}+\frac{P \beta}{4 \sigma^{2}} \Phi_{\mu / \hat{\mu}, k}\right|^{-1}
$$

where $\boldsymbol{S}=\operatorname{diag}\left(s_{1}, \ldots, s_{k}\right)$ and $\hat{\boldsymbol{S}}=\operatorname{diag}\left(\hat{s}_{1}, \ldots, \hat{s}_{k}\right)$ denote the constellation signals from $\mathcal{S}$ transmitted on the subcarrier- $k$, and $\Phi_{\mu / \hat{\mu}, k}$ is the covariance matrix of $s_{k} \boldsymbol{h}_{k}(\boldsymbol{\mu})-\hat{s}_{k} \boldsymbol{h}_{k}(\hat{\boldsymbol{\mu}})$. In $(27), \beta=1$ and $\beta=\digamma$ for the subband and whole-band transmission, respectively.

\section{MRA DESIGN AND OPTIMIZATION FOR MOSK/MOM}

We next develop two practical MRAs suitable for MoSK/MoM. We consider parasitic coupling based MRA technology where a driven antenna element is coupled with a reconfigurable parasitic layer where the surface geometry of this layer, which consists of metallic pixels interconnected by switches, is modified by means of on-off switching [11], [13], [34]. Using low-cost PIN diode switches with 1-3 volts of actuation voltage and switching time of less than 10 nanoseconds [15] provides a highly fast reconfigurability feature that is suitable for MoSK/MoM requiring very short switching times. The criteria for the design of MRAs for MoSK/MoM scheme differ from those of legacy MRA design in that, in addition to the required radiation pattern directivities, one would need to ensure that the resulting set of achievable radiation patterns are as much uncorrelated from each other as possible. Such a design can be realized by the MRA design methodology reported in [35] where the antenna modes are designed such that certain polarizations and radiation patterns can be achieved. For the MoSK/MoM, we impose additional criteria to determine the set of switch states that can create a set of radiation pattern functions optimizing the outcome of mode selection rules (19) or (21). To this end, here, two different MRA designs capable of producing

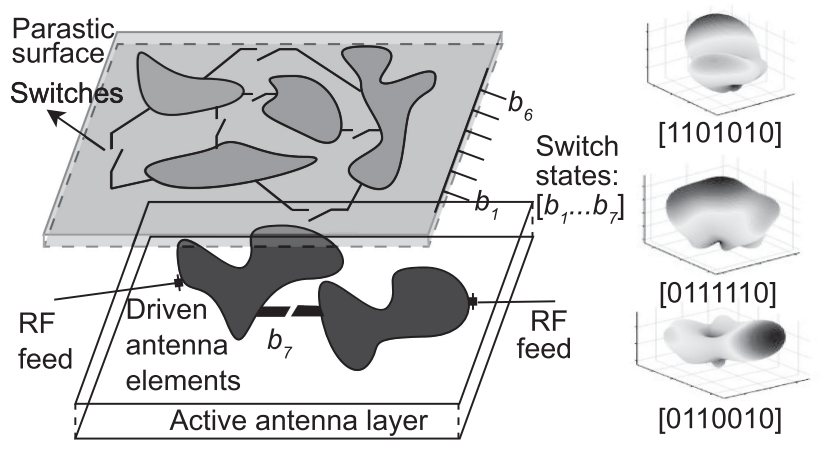

Fig. 3. A generic MRA based on parasitic layer coupling. Both active antenna layer and parasitic layer can be reconfigurable. By setting the state of the switches $\left[b_{1} \ldots b_{7}\right]$, the radiation pattern and the polarization of the antenna can be changed as desired.

a large number of radiation patterns with various shapes and polarizations operating in the $5 \mathrm{GHz}$ band are developed. The differences between the two designs are on the antenna feed circuitry, and in the distribution and the number of parasitic pixel elements used.

\section{A. Parasitic Layer Based MRAs}

An MRA based on a parasitic layer reconfiguration mechanism consists of two main parts, namely, the driven active antenna layer and the reconfigurable parasitic layer (see Fig. 3) The parasitic layer, which is located in the reactive field region of the driven antenna, has a surface typically comprised of electrically small metallic patches, i.e., pixels, interconnected by RF switches. This surface is called a reconfigurable parasitic pixel surface of which geometry can be modified by activating a specific switch configuration. Corresponding to each reconfigurable parasitic pixel surface geometry, there exists varying degree of mutual coupling taking place between the driven and parasitic layers, which is responsible for producing each antenna mode where each mode may have different polarization, radiation pattern and resonance frequency. The parasitic layer based reconfiguration mechanism provides some significant advantages compared to the usual approach, which relies on modifying the geometry of the driven active antenna region [6]. The interconnecting switches along with biasing network placed within the active antenna region pose serious design challenges such as high RF losses and power handling problems resulting from the intense currents in the switches. On the other hand, parasitic layer approach usually keeps the active antenna region unmodified, where reconfiguration capabilities are provided by the reconfigurable pixel layer, which has no physical interconnection to the active antenna region. Thus the problems associated with high RF losses and power handling capabilities are alleviated. Moreover, this approach takes advantage of existing antenna designs and is compatible with a wide variety of antenna architectures. Additionally, it offers advantages in terms of integration capabilities useful for developing sophisticated MRA architectures with high degree of reconfigurability.

As it is well known, multi-antenna transmission schemes such as MoSK/MoM necessitate the correlation between legacy antenna elements to be small (ミ0.6), which can typically be achieved by spatially separating antennas with large enough distances $(\approx \lambda / 2$ with $\lambda$ denoting the wavelength), using 
co-located antennas with patterns that are orthogonal to each other or both [1], [24]. As a single MRA element is capable of producing a large number of antenna modes, we are interested in MRA designs providing highly uncorrelated modes, which has been the main design criteria adopted in developing the two MRA designs explained below. The orthogonality among the antenna modes can be accomplished by using patterns that are different in terms of their polarization properties and the three dimensional geometrical shape of the pattern itself. It is worth noting that the polarization property of a given antenna mode may change significantly over its radiation pattern $\vec{f}(\theta, \phi, \mu)$. At the same time providing good impedance matching along with high realized gain values for all the modes of interest at the design frequency is required. Both our MRA designs are capable of providing large number of modes with the aforementioned properties with trade-offs in the complexity of parasitic layer and the RF feed circuitry driving the active antenna.

\section{B. MRA Design I: Simple Driven Patch Antenna With $3 \times 3$ Parasitic Pixels}

This MRA design, Design I, consists of a driven patch antenna fed by a coaxial cable and a parasitic layer of which upper surface, reconfigurable parasitic pixel surface, has a grid of $3 \times 3$ electrically small squared-shaped metallic pixels. The schematic of the MRA with critical design parameters are given in Fig. 4(a). Notice that an air layer between driven antenna and parasitic layers is employed taking advantage of its lowloss nature. This design is similar to the one presented in [36] with the main difference being in the feed circuitry (coaxial vs. microstrip) and the frequency band of operation ( $5 \mathrm{GHz}$ vs. $2.4 \mathrm{GHz}$ ). Also, while in [36], the MRA is designed solely to provide beam-steering capabilities with the highest realized gain values in few specific directions, the main design criteria in this work is to provide as large number of modes as possible with the highest degree of decorrelation among them. For example, while the design problem for the MRA in [36] is to determine only a few modes, the current design problem is to determine several tens of modes. The MRA designs producing the desired modes is performed by the full-wave electromagnetic analysis tool HFSS [22], used in conjunction with multi-objective genetic algorithm (GA) optimization. Design I compared to the next MRA design, Design II, given below, relies on a more complex parasitic layer, i.e., $3 \times 3$ pixels with 12 interconnecting RF switches, while employing a much simpler RF feed circuitry, i.e., single coaxial cable, in producing the desired modes with radiation patterns of various $3-\mathrm{D}$ shapes and polarizations. We note that this MRA design is based on the one in [36], which was fabricated and measured with good agreement between simulations and measurements. Switch integration, biasing circuitry and fabrication processes are similar to the one given in [36], and thereby, omitted here.

\section{MRA Design II: Polarization Agile Driven Patch With $2 \times 4$ Pixels}

The overall architecture of this MRA design, Design II, is similar to that of Design I (see Fig. 4(b)). This design employs a simpler reconfigurable parasitic pixel surface, where $2 \times 4$ pixels with only 6 interconnecting switches are used. However, to obtain a similar number of highly decorrelated modes as gen-

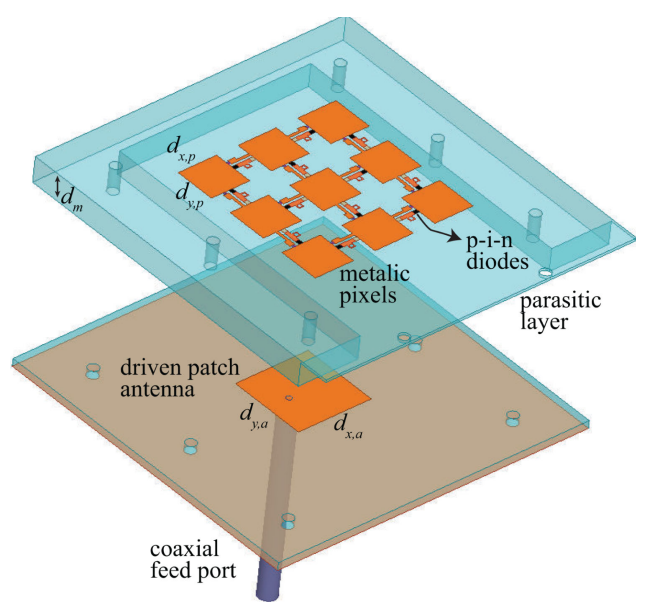

(a)

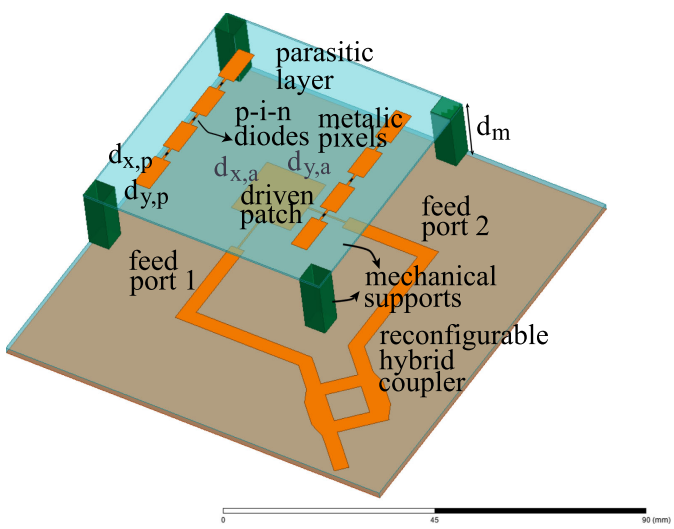

(b)

Fig. 4. Parasitic coupling based MRA designs for $5 \mathrm{GHz}$ band, $d_{x, p}$ and $d_{y, p}$ denote the size of rectangular metallic pixels, $d_{x, a}$ and $d_{y, a}$ denote the size of driven patch antenna. (a) Design I: $3 \times 3$ pixel elements interconnected with 12 switches, $d_{m}$ denote the separation between the pixel surface and the driven patch antenna. This antenna can attain 4096 modes. (b) Design II: Dualpolarized MRA capable of 5 different polarizations including linear $(x-, y-$, the slanted linear), right hand and left hand circular polarizations. This antenna can attain 320 different modes.

erated by Design I, Design II needs to employ a more complex RF feed circuitry. This consists of two RF ports used in conjunction with a reconfigurable directional coupler circuit [37], which enables the driven antenna to generate two orthogonal linear polarizations $(x-$ and $y-)$, slanted linear polarization, and two orthogonal circular polarizations (right-hand CP and lefthand $\mathrm{CP}$ ). Therefore, with a simple parasitic layer, the desired decorrelated modes can be obtained. The complexity is tradedoff between parasitic layer and RF feed circuitry in Designs I and II. Notice that in Design I, the coaxial-fed driven patch antenna only produces a single linear polarization. However, the complexity introduced in the parasitic layer still enables us to obtain large number of decorrelated modes due to different polarizations and geometrical shapes of the radiation pattern.

Depending on the targeted modes of operations and other constraints required by the specific application, Design I or Design II can alternatively be used. In general, the complexity introduced at the RF feed circuitry would result in more system level cost and reduced gain due to RF losses as compared to the case where the complexity was introduced at the parasitic 
TABLE II

CRITICAL DESIGN PARAMETERS (IN mm)

\begin{tabular}{|c|c|c|c|c|}
\hline & Variable & Value & Variable & Value \\
\hline Design I & $\left(d_{x, a}, d_{y, a}\right)$ & $(15.2,15.2)$ & $\left(d_{x, p}, d_{y, p}\right)$ & $(8,8)$ \\
\hline Design II & $\left(d_{x, a}, d_{y, a}\right)$ & $(15,15)$ & $\left(d_{x, p}, d_{y, p}\right)$ & $(8.75,4)$ \\
\hline $\begin{array}{l}\text { Design I } \\
\text { Design II }\end{array}$ & $\frac{d_{m}}{d_{m}}$ & $\frac{5}{7}$ & & \\
\hline
\end{tabular}

layer. It is also worth noting that if the design goal is limited to beam-steering capability in a few specific directions, then the Design II with a simple single RF feed (i.e., a single microstrip feed) can be suitable, and thus provides simplicity advantages compared to Design I.

\section{Critical Design Parameters for MoSK/MoM}

The values of critical design parameters for Design I and II are presented in Table II. The simulated reflection coefficients of various modes of operation with common bandwidths (BWs) for both MRAs are given in Fig. 5. Maintaining a common BW while configuring the radiation patterns of an MRA is one of the most critical design tasks. As seen from Figs. 5(a) and 5(b), the intersections of the individual reflection coefficients indicate a common BW of $130 \mathrm{MHz}$ and $90 \mathrm{MHz}$ for MRA Design I and MRA Design II, respectively.

Next, let us analyze the pattern correlation performance as defined in (2). Let $\boldsymbol{\alpha}_{\mu_{r}}$ denote the synthesis coefficients of the fixed element E-field pattern $\vec{f}\left(\theta, \phi, \mu_{r}\right)$, of the receive antenna. The resulting set of MRA modes shall have E-field patterns such that (i) $\left|\boldsymbol{\alpha}_{\mu_{i}}^{H} \boldsymbol{\alpha}_{\mu_{r}}\right| \geq p_{t h}, \forall i$, so that polarization mismatch, $p_{t h}$, is sufficiently low, and (ii) $\left|\boldsymbol{\alpha}_{\mu_{i}}^{H} \boldsymbol{\alpha}_{\mu_{j}}\right| \leq \rho$ so that the channel spatial signatures to differentiate the transmitted MoSK symbols are as diverse as possible. Using a full-wave EM analysis tool [22], several modes of operation are obtained for each of the MRA designs proposed above. For Design I, there exist 4096 available modes, therefore a GA based search is performed to determine appropriate modes for MoSK modulation. Design II allows us to generate 5 different set of polarizations from the driven antenna. Therefore, there exist $2^{v}(v=6$ being the number of interconnecting switches) different E-field shapes for each polarization type, which results in a total of 320 different modes of operations.

The empirical cumulative distribution functions (CDF) of the pairwise normalized pattern correlation between the antenna modes achieved by Designs I and II are depicted in Fig. 6(a). The figure depicts the results for two approaches: (i) random selection of 32 modes out of all available modes, and (ii) selection of 32 modes minimizing the sum of pairwise pattern correlation values. Thus, in each case, 496 different mode pairs exist. It is seen that the optimized modes of both designs include antenna modes that can attain small correlations. For Design I, a GA based optimization is performed to determine the optimized modes out of all 4096 modes available. Design II has only 320 different modes of operation and a random search based optimization is performed to obtain the best 32 modes. With Design II, smaller pattern correlations have been observed even though the number of mode pairs with lower correlations is less than that achieved by Design I. For example, from the CDF curves indicating the number of mode pairs with a correlation coefficient less than some threshold, it is seen that, about $60 \%$ of the associated mode pairs (e.g., 300 mode pairs) have

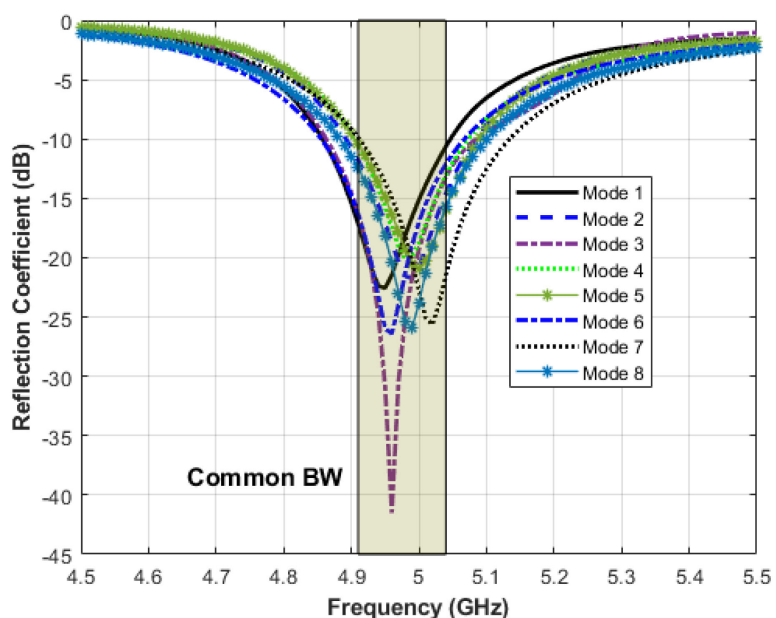

(a)

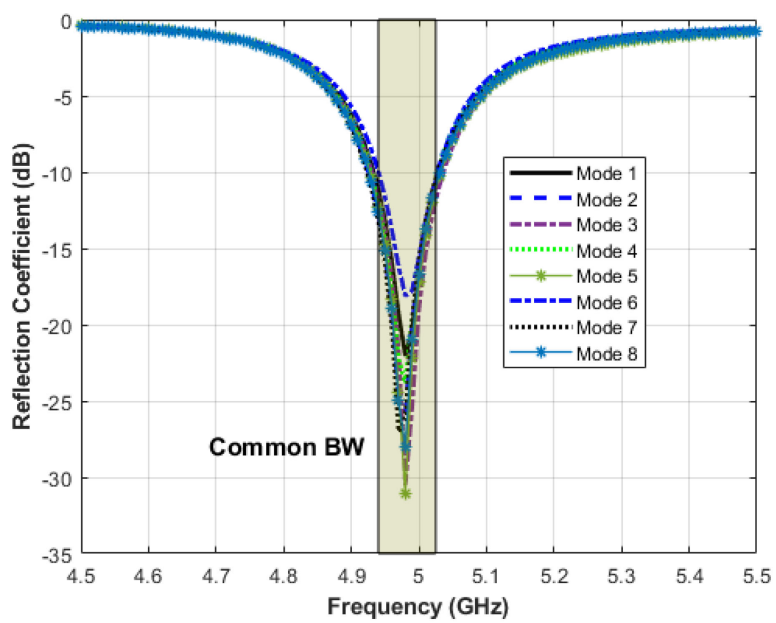

(b)

Fig. 5. Simulated reflection coefficients of the MRAs. (a) Design I (b) Design II.

correlation magnitudes of less than 0.6 for Design I, while it is about $48 \%$ (e.g., 240 pairs) for Design II.

The relationship between the pairwise-pattern correlation and the resulting performance difference based on (11) is illustrated in Fig. 6(b). Here, the underlying set of best $L_{a} \in\{2,4,8\}$ modes for MoSK are selected from the 32 optimized patterns such that the sum of pairwise pattern correlation values is minimized. For $L_{a}=2,4$, an exhaustive search is performed, while for $L_{a}=8$, a random search based optimization is employed. In the figure, $\rho_{w}$ denotes the pairwise pattern correlation with the highest magnitude among all $\left(\begin{array}{c}L_{a} \\ 2\end{array}\right)$ pairs. The values of $\zeta$ are obtained for an urban-micro B1 channel [38] (please see Section VI for simulation setup). It is seen from the $\zeta$ values that both MRA designs provide $L_{a}=2,4$ modes with significantly improved error rate performance compared to the legacy SSK scheme employing dipole arrays. For $L_{a}=8$, the union bound predicts a comparable MoSK performance with legacy SSK. With 8 modes, MRA I can achieve $\zeta=0.4 \mathrm{~dB}$, i.e., implying a slightly better performance, whereas for MRA 2, we have $\zeta=-3.1$, i.e., a slightly worse performance compared to the legacy SSK. Nevertheless, the MoSK scheme will require 


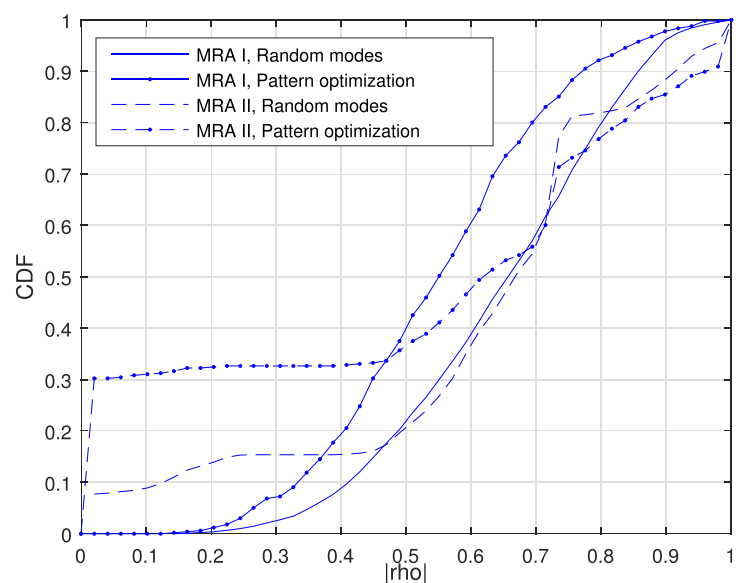

(a)

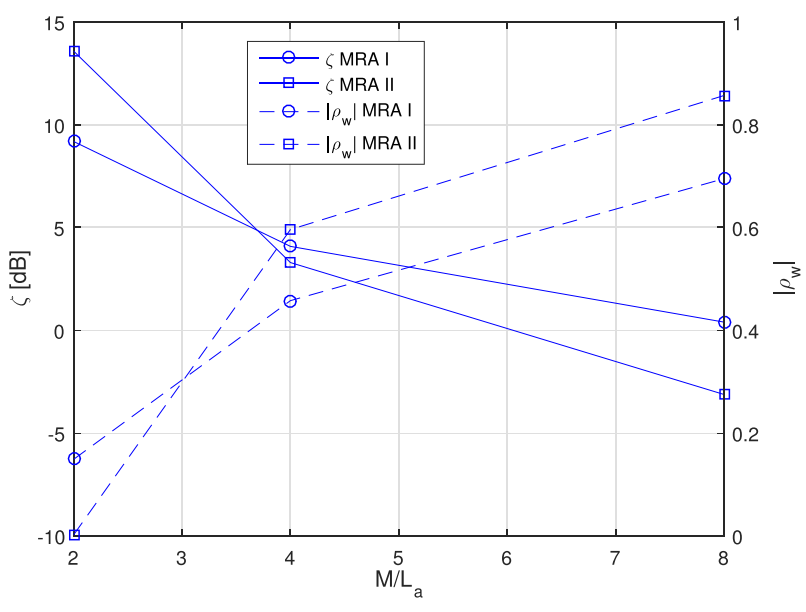

(b)

Fig. 6. (a) The CDF of normalized correlation coefficients for MRA E-field patterns for Designs I and II. Correlation performances for two cases are depicted: (1) random design, (2) optimized design for MoSK according to the minimization of sum of pattern correlation between the selected modes for MoSK. For both designs, 32 modes are determined for MoSK. (b) Relation between the pairwise pattern correlation $\left(\rho_{w}\right), M / L_{a}$, and $\zeta$ [from union bound analysis in (11)].

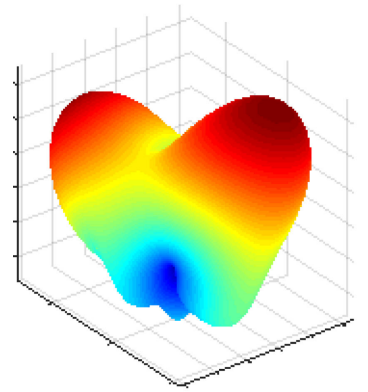

(a)

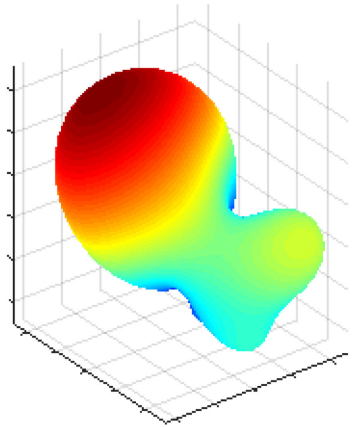

(b)

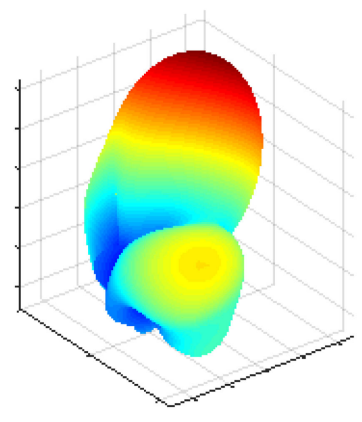

(c)

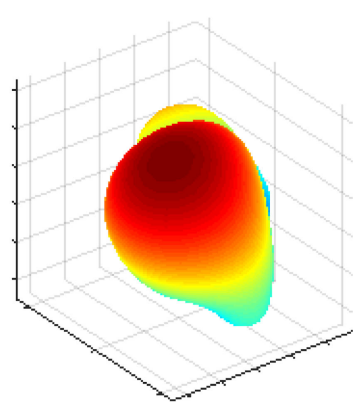

(d)

Fig. 7. Radiation patterns for 4 modes corresponding to $L_{a}=4$ case in 6 (b) for MRA I.

significantly lower hardware complexity due to use of a single reconfigurable antenna element instead of $M=8$ elements along with the complicated RF switching circuitry.

The normalized radiation patterns for the $L_{a}=4$ case in 6(b) are depicted in Figs. 7 and 8 for MRA I and II, respectively. For MRA I, the low-correlation among radiation patterns are mainly achieved in the spatial domain. For MRA II, the pattern in Fig. 8(a) is $+x$ polarized while the ones in (b)-(d) are $+y$ polarized, thereby, resulting in very low correlation with them. The low correlation among the three $+y$ polarized modes in Fig. 8 is achieved in the spatial domain.

\section{NUMERICAL RESULTS}

We next study the performance of proposed MoSK/MoM schemes with both MRA designs via numerical examples. The transmitter for legacy SSK/SM and the proposed MoSK/MoM schemes along with the receiver system that is common to both transmission schemes are illustrated in Figs. 9(a), 9(b) and 9(c), respectively. In case of legacy SSK/SM, a MIMO antenna with $M$ dipole elements separated by $\lambda / 2$ are assumed (linear array) for which the antenna module occupies an area of $(M-1) \lambda / 2 \times \lambda / 2$. For the proposed MoSK/MoM schemes, a single transmit MRA element (with a dimension of $\lambda / 2 \times \lambda / 2$ ) with $L_{\mu}$ modes is considered and the radiation patterns are obtained by full-wave EM analyses [22]. The values of the underlying parameters are specified for each example separately. The MIMO receiver has a 2 - element crossed-dipole array with $\lambda / 2$ interelement spacing, e.g., two $\pm 45^{\circ}$-slanted dipoles amounting to $N=4$ dipole elements. As seen in Fig. 9(a), a SPMT switch is required to switch among the dipole elements. For the MoSK/MoM scheme, this SPMT is removed and PIN diode switch control is employed to switch among the different MRA modes. The channel is modeled using a 3D double directional MIMO channel [39], [40] modified for the underlying MR-MIMO systems. For a fair comparison, the channel power averaged over all antenna modes is normalized to unity, e.g., $\frac{1}{L_{\mu}} \sum_{\mu=1}^{L_{\mu}} E\left(\left|h_{m}(\mu)\right|^{2}\right)=1$, and the average signal to noise ratio is defined as $P / \sigma^{2}$.

\section{A. PEP Analysis and MRA Design for MoSK}

Let us first study the theoretical analysis by means of numerical calculations. Since the MC PEP analysis follows similarly as the one for SC case, for brevity, we limit the discussion here to the SC case. In Figs. 10(a) and 10(b), the PEP and the symbol error probability results are depicted for MoSK and SSK schemes. For both SSK and MoSK schemes, Fig. 10(a) shows 


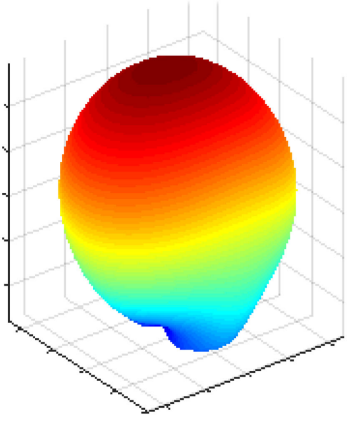

(a)

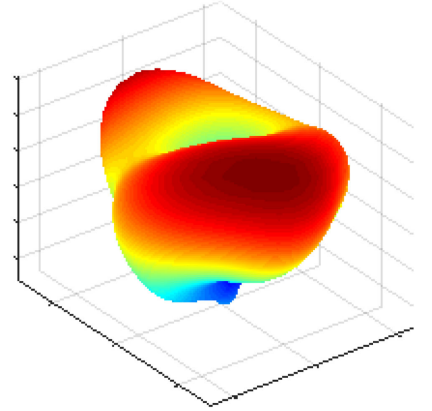

(b)

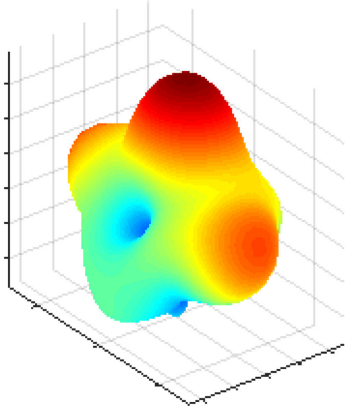

(c)

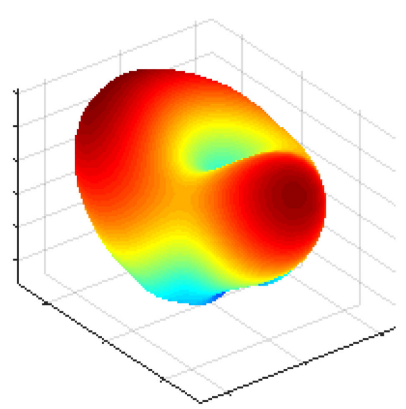

(d)

Fig. 8. Radiation patterns for 4 modes corresponding to $L_{a}=4$ case in 6 (b) for MRA II. The pattern in (a) is $+x$ polarized while the ones in (b) $-(\mathrm{d})$ are $+y$ polarized, hence, resulting in very low correlation with them. The low correlation among the three $+y$ polarized modes is achieved in the spatial domain.

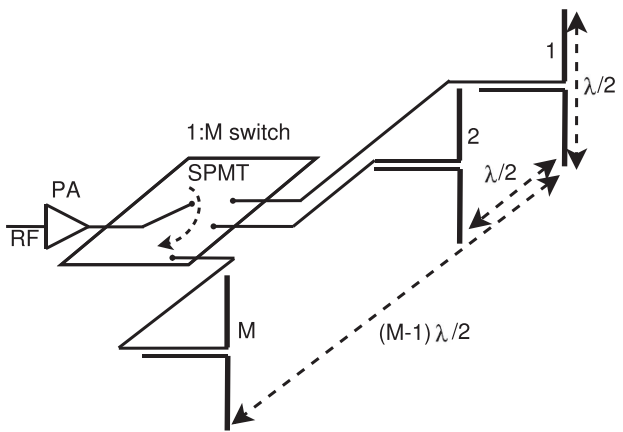

(a)

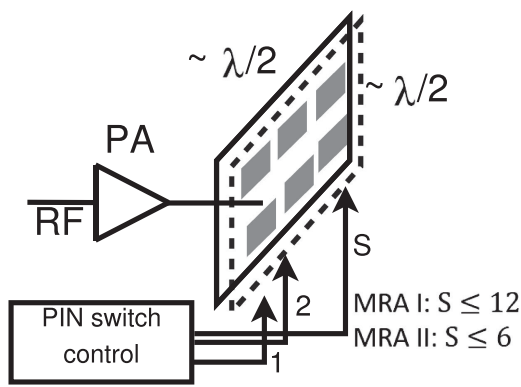

(b)

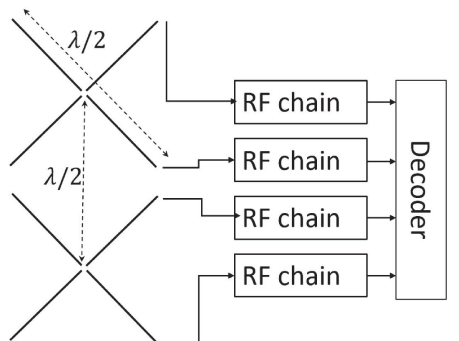

(c)

Fig. 9. (a) Legacy SSK/SM transmitter with $M$ half-wavelength dipole antennas, (b) Proposed MoSK/MoM transmitter with single MRA element, and (c) Receiver with $2 \times 1$ linear array of $\pm 45^{\circ}$ cross-dipoles (common for SSK/SM and MoSK/MoM schemes.)

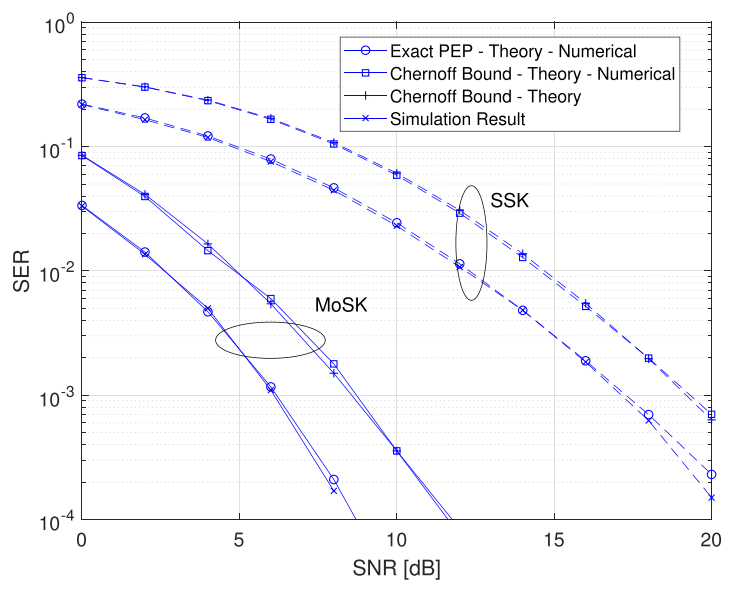

(a)

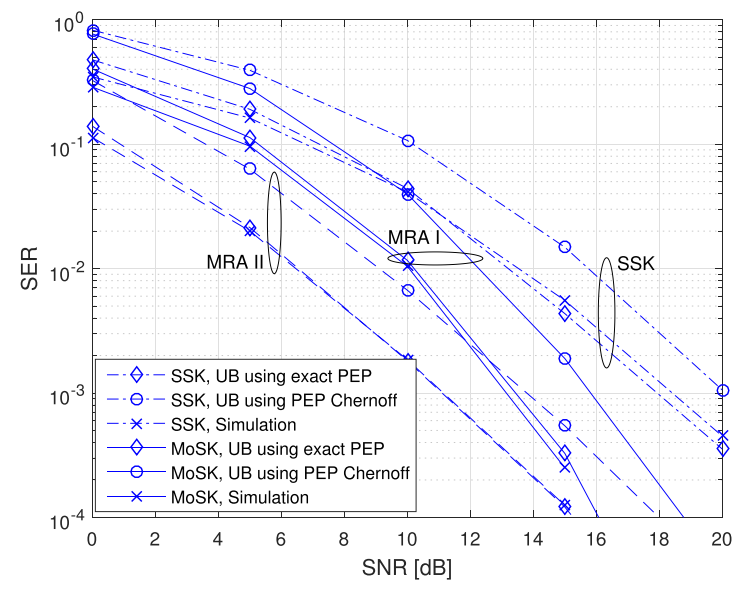

(b)

Fig. 10. (a) Theoretical and simulation results for PEP when SSK and MoSK are employed. MRA Design I is assumed. (b) Union bound and simulation results with $r=\log _{2}(4)$ bits/channel use for SSK and MoSK. Results for MRA Designs I and II are presented. For comparison purposes, SSK performance is also included.

(i) the exact PEP obtained from integration of $Q(\cdot)$ in (28) (in the Appendix) over the fading statistics, (ii) the Chernoff bound numerically evaluated using (30), (iii) the Chernoff bound formula obtained in (31), and (iv) the PEP from simulations. MRA Design I is used at the transmitter. For SSK, $M=2$ antennas are employed while for MoSK, $L_{a}=2$ modes among a pool of 16 are employed, and for each scheme, $10^{5}$ channel samples are generated. It is seen that the PEP with simulation results and the theoretical calculations agree very well. We note that the performance of both schemes depends on the spatial correlation 


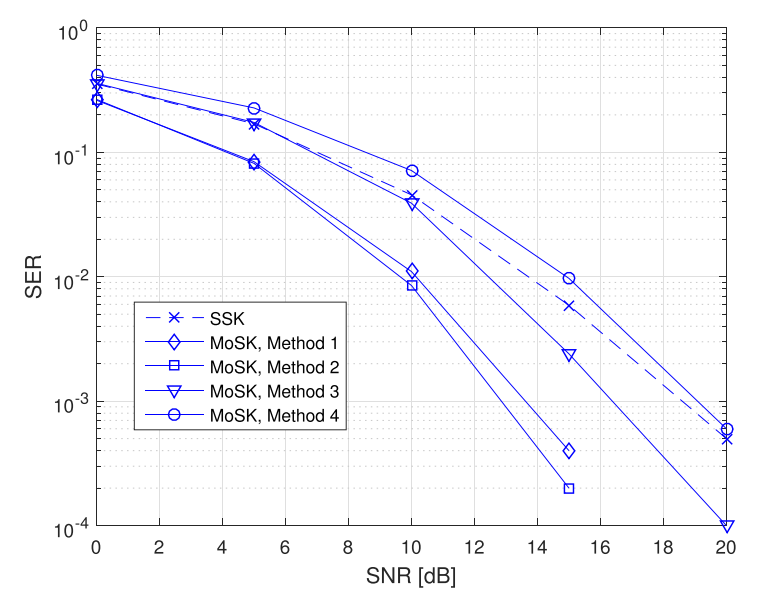

(a)

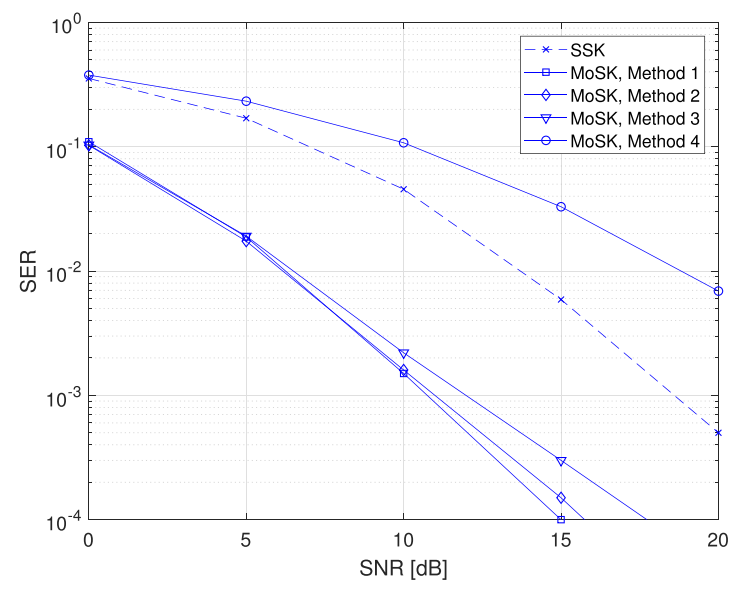

(b)

Fig. 11. MoSK performance with different design methods for (a) MRA Design I and (b) MRA Design II.

between the associated channels. In this example, the selected mode pair for MoSK results in significantly lower correlation compared to that of the SSK scheme with $\lambda / 2$ interelement spacing between the antenna elements, thus achieving a significant SNR gain.

In Fig. 10(b), the union bound results are provided for SSK with $M=4$ transmit antennas, and for MoSK with $L_{\mu}=4$. For comparison purposes, we also plot the symbol error rates (SERs) obtained from simulations. The results for both MRA designs are depicted. It is again observed that the theoretically evaluated expressions are in line with the symbol error probabilities obtained from simulations. It is also seen that the union bound using the $Q$-function based PEPs are very tight. The performance-complexity trade-off is also clear from the SER results for MRA Designs I and II. We also note that, for low SNR values, the SER difference between SSK and MoSK is small. This is due to the fact that, at small SNRs, the thermal noise (AWGN) is more dominant factor for the system performance, and hence, we see a comparatively smaller performance improvement. For higher SNRs, channel correlation becomes the dominant factor and the diverse set of MRA modes can be utilized to increase the channel diversity.

The impact of mode set construction techniques (e.g., in Section C) on the system performance is shown in Figs. 11(a) and 11(b), for Designs I and II, respectively, when $L_{a}=4$ modes are employed. The methods mentioned in the legend of the figure are as follows:

- Method 1: Random search using the channel covariance matrices (according to (13)),

- Method 2: Iterative method based on the knowledge of the channel covariance matrices (according to (14)),

- Method 3: Random search based on the pattern covariance matrix (according to (19)),

- Method 4: Iterative method based on the pattern covariance matrix (according to (21)).

For both examples, it is seen that the knowledge of channel covariance matrices help construct a mode set with superior MoSK performance. What is more, in this example, random search with a low number of iterations, e.g., 1000 iterations, is able to find a suitable mode set even though the search space is very large. The iterative approach utilizing the channel covariance matrix (Method 2) with only $L_{a}$ iterations can determine the mode sets with similar symbol error performance. In these figures, the SSK performance with legacy antennas is also shown. The mode selection relying only on the pattern correlations can also determine the modes with which MoSK can perform comparable to legacy SSK schemes, e.g., Method 3. If the iterative approach is performed using the pattern correlations (Method 4), MRA I achieves a slightly worse performance while for MRA II, it may not converge to a good solution. This is mainly due to the fact that MRA I can achieve a larger diverse set of radiation patterns as compared to the MRA II. We note that the main advantage with the proposed methods against the legacy SSK is that they determine the MRA modes that create sufficiently decorrelated spatial channels, which is required for improved detection. In addition, the proposed scheme always employs a single MRA element with a single RF chain, and hence, avoiding the RF design issues present for SSK schemes.

In the following subsections, we investigate the performance of MoSK/MoM schemes, and compare their performance with SSK/SM. Since Method 2 is superior to other design approaches and it is computationally less demanding, we employ this method to select the MRA modes for MoSK and MoM schemes. Note that while Method 2 requires the knowledge of covariance matrix, this is not a big issue as one can estimate the covariance matrix using the estimated channel state information. Here, we simply assume that the channel covariance information is available to the transmitter. In this work it is assumed that the scatterers are quasistatic, that is, they remain the same over a relatively longer period of time compared to the symbol duration, while the temporal variations due to user velocity are more pronounced. We note that, for a quasistatic propagation environment, the covariance matrices do not vary significantly over time [24] and thus, one will incur low estimation overhead (see, for example, [25]).

\section{B. Performance With Narrowband Transmission}

Figs. 12(a) and 12(b) demonstrate the performance improvements offered by the proposed MoSK and MoM schemes over the legacy SSK and SM, respectively. For a fair comparison, the data rates of SSK and MoSK (and similarly, SM and MoM) are chosen to be the same, i.e., the number of transmit antennas, $M$, is equal to the number of antenna modes, $L_{a}$, with a transmission rate of $R=\log _{2}\left(L_{a}\right)$ bits/channel use. Simulation 


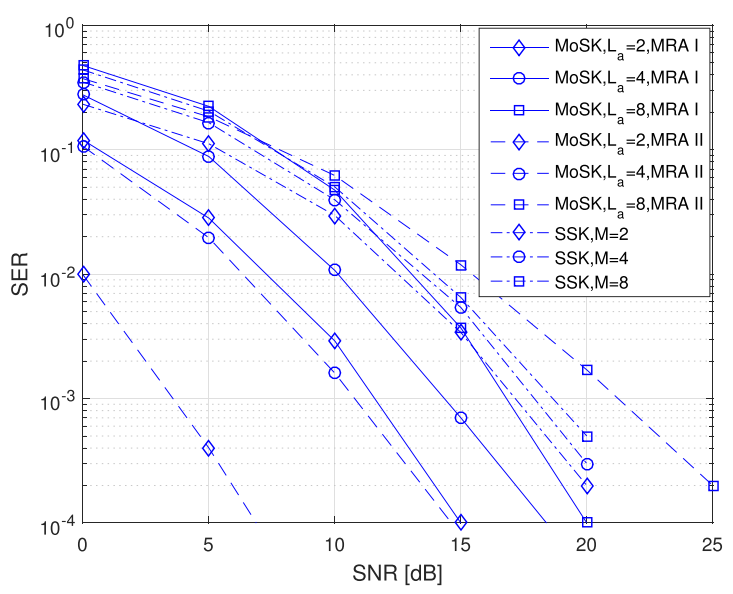

(a)

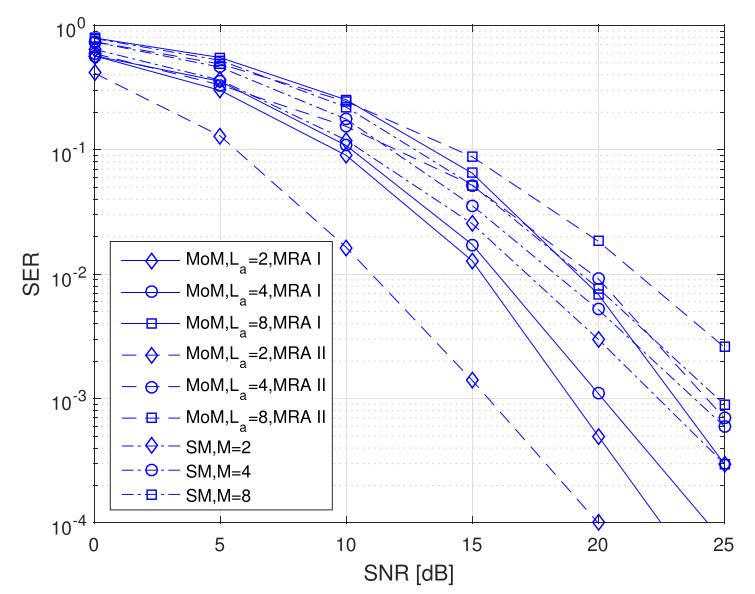

(b)

Fig. 12. Symbol error rate comparison for (a) legacy SSK versus MoSK and (b) legacy SM versus MoM.

results are provided for both MRA designs (Designs I/II). In the first example, both Designs I and II have sixteen modes from which the mode selection algorithm selects $L_{a}$ modes. From Figs. 12(a), it is observed that MoSK is superior to SSK for the cases of $R=1$ and 2 bits/channel use for both Designs I and II. For $R=3$ bits/channel, MRA I performs slightly better than the SSK scheme for SNR $>10 \mathrm{~dB}$, while MRA II performs slightly worse. A similar trend for SER is observed where the error performance deteriorates as the $M / L_{a}$ is increased for both cases. For $R=3$, the performance with MoSK and SSK becomes similar, however, note that the MoSK scheme employs a single element as opposed to the SSK scheme which requires 8 antenna elements. The SNR gains are achieved with a significantly less complexity as the excitation of MRA modes is accomplished by controlling the PIN diodes on the parasitic surface with a simple low-power circuitry. In addition, the overhead due to channel covariance estimation is very small since the second order characteristics of the wireless channel is relatively slowly varying. In other words, one can estimate the channel covariance matrix for the underlying modes and perform one-time mode set selection after which the same set of modes can be employed for the rest of the transmissions.

Performance comparisons between the legacy SM and the proposed MoM schemes are provided in Figs. 12(b) for Designs I and II, respectively. Here, a rectangular 16-QAM constellation is employed. For legacy SM, we present the SER for cases of $M=2,4$ and 8 antennas and for the corresponding MoM schemes we employ MRAs with $L_{a}=2,4$ and 8 modes, respectively. The transmission rate is $\log _{2}(X)+\log _{2}(16)$ bits/channel use where $X=M$ for SM and $X=L_{a}$ for MoM. It is observed that MoM achieves a better or comparable error performance compared to legacy SM. For example, MRA I provides $7 \mathrm{~dB}$ and $2 \mathrm{~dB}$ gain for $L_{a}=2$ and 8 , respectively, at an SER of $10^{-3}$. With MRA II, employing 8 modes for this underlying channels requires $2.5 \mathrm{~dB}$ more SNR to achieve the same SER with SM with $M=8$ antenna elements. Hence, a trade-off between the rate (e.g., number of modes activated for MoM) and error performance is available for MRA II.

\section{Performance With Wideband Transmission}

Figs. 13(a) and 13(b) depict SER comparisons of SSK versus MoSK, and SM versus MoM, for wideband transmission sce- narios, respectively, with Design I. For comparison purposes, the SER curves assuming SC for the cases of $M=L_{a}=8$ are also included. For the wideband case, an MC scheme assuming $K=4$ subcarriers is assumed. Similar to the case of narrowband transmission, the modes in the transmitter are chosen using the Method 2 as described in Section A. From the simulation results it is observed that increasing the number of subcarriers to $K=4$ improves the performance for all the cases as expected. The gains are approximately $10 \mathrm{~dB}$ for both MoSK and SSK schemes, with MoSK being superior to SSK as before. This additional gain can be used to increase the data rate by increasing the number of transmitting modes for the MoSK scheme, as opposed to the need for a larger number of antennas for the SSK scheme which may not be feasible as the number of antennas is typically fixed by deployment constraints or design. We demonstrate the SER for the cases of $L_{a}=16$ and 32 for the MoSK schemes. With only 4 subcarriers, the MRA system activating 32 modes achieves a comparable SER performance to the single carrier case with 8 modes. These results represent a data rate increase from 3 bits/mode to 5 bits/mode, e.g., $66 \%$ improvement in throughput, with a similar performance. Note also that one would need 32 antenna elements to achieve the same spectral efficiency with SSK, which may be prohibitive due to the need for antenna hardware modifications. Considering the impact of switching time $\left(T_{s w}\right)$, the MoSK/MoM with multicarrier transmission can significantly outperform the throughput of the legacy SSK system since it achieves the improvement without any increase in the number of PIN diode switches whereas legacy SSK system would suffer from increased SPMT switching time delays.

In Fig. 13(b), we demonstrate that similar results are obtained in case of SM and MoM for Design I using $K=4$ subcarriers. In this case, even though the relative gains with $\mathrm{MC}$ transmission are less pronounced compared to the those for the SSK/MoSK scheme, MoM with up to 32 modes can achieve similar SERs with the single carrier SSK with 8 antennas. Once again, significant spectral efficiency improvements can be attained with only a slight increase in computational complexity while an equivalent SSK/SM would require a larger number of antenna elements to achieve similar data rates.

It is seen from the above examples that the MRA design and optimization can significantly improve the MoSK and MoM performance over the legacy SSK/SM schemes while removing 


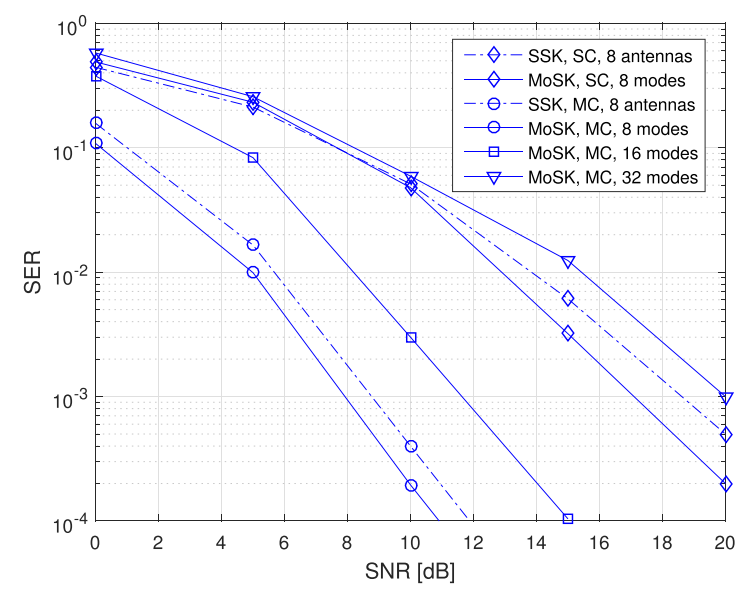

(a)

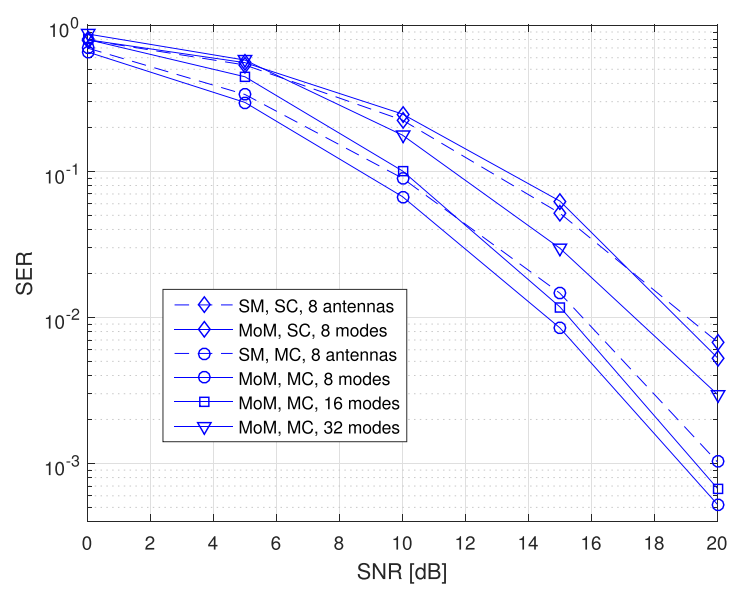

(b)

Fig. 13. (a) Legacy SSK versus MoSK with 4 subcarrier. (b) SM and MoM performance, for 4 subcarriers, 16-QAM.

the need for antenna switching circuitry in the RF path and introducing a smaller number of PIN diode switches in the parasitic layer of the MRAs. The ohmic losses of the PIN diodes used are $0.1 \mathrm{~dB}$. Therefore, if the number of diodes used is not excessively large, which is one of the optimization criteria in designing the MRAs, the diode losses do not play any significant role on the system performance.

\section{CONCLUDING REMARKS}

In this paper, we have proposed employing MRAs with dynamically changeable modes for SSK/SM based transmission, which are referred to as mode-shift keying (MoSK) and mode modulation (MoM) schemes, and demonstrated that the proposed schemes can accommodate higher data rates and offer improved error probabilities compared to legacy SSK and SM. We developed two MRA designs that are optimized for MoSK/MoM based transmissions. By utilizing the radiation pattern sets, i.e., modes, resulting in low correlations among the spatial channels, which is not possible with the usual SSK/SM, decoding errors can be reduced. We have shown that the additional flexibility offered by the modes of the MRAs can improve the system performance with a significant reduction in complexity. Moreover, simulation results show that MoSK/MoM with MC based transmission can achieve lower symbol error rates for a given transmit SNR compared to SC based MoSK/MoM. The flexibility of MRA elements allows for dynamically changing and optimizing the number of operational modes and hence creates a very efficient spatial modulation scheme.

In this work, we have focused on the ideal performance for both SSK/SM and MoSK/MoM schemes. Extension of the analysis to evaluate the impact of the channel estimation errors on the performance may be pursued as a further study. Alternative MRA architectures that can result in radiation patterns with lower pattern correlations can also be investigated. In addition, a system level design approach where MIMO transmission schemes and MRAs are jointly designed open up other interesting research directions. Finally, we note that these ideas can also be useful for general MIMO transmission where the wireless system can switch between different MIMO schemes including precoding, spatial multiplexing, space-time coding in addition to the SSK/SM type transmissions.

\section{APPENDIX \\ PEP BOUND FOR MOSK AND MOM}

For the MoSK scheme, assuming that the channel is fixed for a transmitted waveform $s$ normalized to unit power, the PEP and the resulting Chernoff bound for maximum likelihood decoding of the mode index can be expressed, respectively, as

$$
\begin{aligned}
P\left(\mu \rightarrow \hat{\mu} \mid \boldsymbol{h}_{m}(\mu), \boldsymbol{h}_{m}(\hat{\mu})\right)= & Q\left(\sqrt{\frac{P\left\|\Delta_{m}(\mu, \hat{\mu})\right\|_{F}^{2}}{2 \sigma^{2}}}\right) \\
& \leq \exp \left(-\frac{P}{4 \sigma^{2}}\left\|\Delta_{m}(\mu, \hat{\mu})\right\|_{F}^{2}\right)
\end{aligned}
$$

where $\Delta_{m}(\mu, \hat{\mu})=\boldsymbol{h}_{m}(\mu)-\boldsymbol{h}_{m}(\hat{\mu})$ and $\|\cdot\|_{F}$ denotes the Frobenius norm. For the jointly Gaussian $\boldsymbol{h}_{m}(\mu)$ and $\boldsymbol{h}_{m}(\hat{\mu})$, the channel vector difference $\boldsymbol{h}_{m}(\mu)-\boldsymbol{h}_{m}(\hat{\mu})$ becomes a zeromean complex Gaussian vector with covariance matrix

$$
\Upsilon_{\mu / \hat{\mu}}=\Upsilon_{\mu, \mu}+\Upsilon_{\hat{\mu}, \hat{\mu}}-\Upsilon_{\mu, \hat{\mu}}-\Upsilon_{\hat{\mu}, \mu}
$$

By proper design of the underlying antenna modes $(\mu, \hat{\mu})$, and assuming rich scattering, this covariance matrix can be made full-rank, i.e., $\operatorname{rank}\left(\Upsilon_{\mu / \hat{\mu}}\right)=N$. Using the characteristic function of $\|\boldsymbol{x}-\boldsymbol{y}\|_{F}^{2}$ derived in [27], the PEP averaged over the fading channel for the MoSK can then be obtained as

$$
\begin{aligned}
\bar{P}(\mu \rightarrow \hat{\mu}) & \leq E_{\boldsymbol{h}_{m}(\mu), \boldsymbol{h}_{m}(\hat{\mu})}\left\{\exp \left(-\frac{P}{4 \sigma^{2}}\left\|\Delta_{m}(\mu, \hat{\mu})\right\|_{F}^{2}\right)\right\} \\
& \leq\left|I_{N}+\frac{P}{4 \sigma^{2}} \Upsilon_{\mu / \hat{\mu}}\right|^{-1} .
\end{aligned}
$$

We note that this result is similar to that of space-time coding transmission where the code-word difference matrix appearing in the case of space-time coding is replaced by the covariance matrix of the channel difference [26]. Let $\mathcal{M}_{a} \subseteq \mathcal{M}$ denote a set of $L_{a} \leq L_{\mu}$ modes selected from all available modes $\mathcal{M}$. For the high-SNR regime, a union bound on the average error 
probability for mode group $\mathcal{M}_{a}$ can be obtained as

$$
P\left(\mathcal{M}_{a}\right) \leq\left(\frac{P}{4 \sigma^{2}}\right)^{-N} \frac{1}{L_{a}} \sum_{\substack{\hat{\mu} \neq \mu \\ \mu, \hat{\mu} \in \mathcal{M}_{a}}} d(\mu, \hat{\mu})^{-1}
$$

where $d(\mu, \hat{\mu})=\lambda_{1} \cdot \lambda_{2} \cdots \lambda_{N}$, and $\lambda_{i}>0$ are the eigenvalues of $\Upsilon_{\mu / \hat{\mu}}$. Note that a similar union bound can be obtained for SSK scheme where $d(\mu, \hat{\mu})$ is replaced by $d(m, \hat{m})=$ $\omega_{1} \cdot \omega_{2} \ldots \omega_{N}$ with $\omega_{i}>0$ denoting the eigenvalues of $\Psi_{m / \hat{m}}$ in (9), and $L_{a}$ by $M$.

For the MoM, a similar analysis can be performed to find the PEP bound for the MLD of $(\mu, s)$ as $(\hat{\mu}, \hat{s})$ as

$$
\bar{P}(\mu \rightarrow \hat{\mu}, s \rightarrow \hat{s}) \leq\left|I_{N}+\frac{P}{4 \sigma^{2}} \Phi_{\mu / \hat{\mu}}\right|^{-1}
$$

where $\Phi_{\mu / \hat{\mu}}=|s|^{2} \Upsilon_{\mu, \mu}+|\hat{s}|^{2} \Upsilon_{\hat{\mu}, \hat{\mu}}-s \hat{s}^{*} \Upsilon_{\mu, \hat{\mu}}-s^{*} \hat{s} \Upsilon_{\hat{\mu}, \mu}$.

\section{REFERENCES}

[1] M. D. Renzo and H. Haas, "Space shift keying (SSK) MIMO over correlated Rician fading channels: Performance analysis and a new method for transmit-diversity," IEEE Trans. Commun., vol. 59, no. 1, pp. 116-129, Jan. 2011.

[2] J. Jeganathan, A. Ghrayeb, L. Szczecinski, and A. Ceron, "Space shift keying modulation for MIMO channels," IEEE Trans. Wireless Commun., vol. 8, no. 7, pp. 3692-3703, Jul. 2009.

[3] J. Jeganathan, A. Ghrayeb, and L. Szczecinski, "Generalized space shift keying modulation for MIMO channels," in Proc. IEEE 19th Int. Symp. Pers., Indoor Mobile Radio Commun., Sep. 2008, pp. 1-5.

[4] R. Mesleh, H. Haas, S. Sinanovic, C. W. Ahn, and S. Yun, "Spatial modulation," IEEE Trans. Veh. Technol., vol. 57, no. 4, pp. 2228-2241, Jul. 2008.

[5] M. Di Renzo, H. Haas, A. Ghrayeb, S. Sugiura, and L. Hanzo, "Spatial modulation for generalized MIMO: Challenges, opportunities, and implementation," Proc. IEEE, vol. 102, no. 1, pp. 56-103, Jan. 2014.

[6] B. Cetiner, H. Jafarkhani, J.-Y. Qian, H. J. Yoo, A. Grau, and F. De Flaviis, "Multifunctional reconfigurable MEMS integrated antennas for adaptive MIMO systems," IEEE Commun. Mag., vol. 42, no. 12, pp. 62 70, Dec. 2004.

[7] A. Sayeed and V. Raghavan, "Maximizing MIMO capacity in sparse multipath with reconfigurable antenna arrays," IEEE J. Sel. Topics Signal Process., vol. 1, no. 1, pp. 156-166, Jun. 2007.

[8] D. Piazza, N. Kirsch, A. Forenza, R. Heath, and K. Dandekar, "Design and evaluation of a reconfigurable antenna array for MIMO systems," IEEE Trans. Antennas Propag., vol. 56, no. 3, pp. 869-881, Mar. 2008.

[9] A. Grau, H. Jafarkhani, and F. De Flaviis, "A reconfigurable multiple-input multiple-output communication system," IEEE Trans. Wireless Commun., vol. 7, no. 5, pp. 1719-1733, May 2008.

[10] B. Cetiner, E. Akay, E. Sengul, and E. Ayanoglu, "A MIMO system with multifunctional reconfigurable antennas," IEEE Antennas Wireless Propag. Lett., vol. 5, no. 1, pp. 463-466, Dec. 2006.

[11] B. Cetiner, D. Rodrigo, and L. Jofre, "Reconfigurable antennas utilizing parasitic pixel layers," WO Patent App. PCT/US2012/059,378, Jul. 18, 2013. [Online]. Available: http://www.google.com/patents/ WO2013106106A2?cl=en

[12] Z. Bouida, H. El-Sallabi, A. Ghrayeb, and K. Qaraqe, "Reconfigurable antenna-based space-shift keying (SSK) for MIMO Rician channels," IEEE Trans. Wireless Commun., vol. 15, no. 1, pp. 446-457, Jan. 2016.

[13] X. Yuan et al., "A parasitic layer-based reconfigurable antenna design by multi-objective optimization," IEEE Trans. Antennas Propag., vol. 60, no. 6, pp. 2690-2701, Jun. 2012.

[14] A. K. Khandani, "Media-based modulation: A new approach to wireless transmission," in Proc. IEEE Int. Symp. Inf. Theory, Jul. 2013, pp. 30503054.

[15] D. Pozar, Microwave Engineering, 4th ed. New York, NY, USA: Wiley, 2011.

[16] M. Di Renzo, H. Haas, and P. M. Grant, "Spatial modulation for multipleantenna wireless systems: A survey," IEEE Commun. Mag., vol. 49, no. 12, pp. 182-191, Dec. 2011.
[17] M. Di Renzo and H. Haas, "Bit error probability of SM-MIMO over generalized fading channels," IEEE Trans. Veh. Technol., vol. 61, no. 3, pp. 1124-1144, Mar. 2012.

[18] J. Jeganathan, A. Ghrayeb, and L. Szczecinski, "Spatial modulation: Optimal detection and performance analysis," IEEE Commun. Lett., vol. 12 , no. 8, pp. 545-547, Aug. 2008.

[19] E. Basar, U. Aygolu, E. Panayirci, and H. Poor, "Space-time block coded spatial modulation," IEEE Trans. Commun., vol. 59, no. 3, pp. 823-832, Mar. 2011

[20] S. Sugiura and L. Hanzo, "Single-RF spatial modulation requires singlecarrier transmission: Frequency-domain turbo equalization for dispersive channels," IEEE Trans. Veh. Technol., vol. 64, no. 10, pp. 4870-4875, Oct. 2015.

[21] S. Ganesan, R. Mesleh, H. Ho, C. W. Ahn, and S. Yun, "On the performance of spatial modulation OFDM," in Proc. 40th Asilomar Conf. Signals, Syst. Comput., Oct. 2006, pp. 1825-1829.

[22] ANSYS, "ANSYS HFSS, version 16.0," 2016. [Online]. Available: http://www.ansys.com

[23] A. Kalis, A. Kanatas, and C. Papadias, Parasitic Antenna Arrays for Wireless MIMO Systems. New York, NY, USA: Springer, 2013.

[24] C. Oestges and B. Clerckx, MIMO Wireless Communications: From RealWorld Propagation to Space-Time Code Design. Amsterdam, The Netherlands: Elsevier, 2010.

[25] I. Bahceci, M. Hasan, T. M. Duman, and B. A. Cetiner, "Efficient channel estimation for reconfigurable mimo antennas: Training techniques and performance analysis," IEEE Trans. Wireless Commun., vol. 16, no. 1, pp. 565-580, Jan. 2017.

[26] T. M. Duman and A. Ghrayeb, Coding for MIMO Communication Systems England: John Wiley and Sons, 2007.

[27] R. Mallik, "On multivariate Rayleigh and exponential distributions," IEEE Trans. Inf. Theory, vol. 49, no. 6, pp. 1499-1515, Jun. 2003.

[28] R. Mesleh, O. Hiari, A. Younis, and S. Alouneh, "Transmitter design and hardware considerations for different space modulation techniques," IEEE Trans. Wireless Commun., vol. 16, no. 11, pp. 7512-7522, Nov. 2017.

[29] E. Soujeri and G. Kaddoum, "The impact of antenna switching time on spatial modulation," IEEE Wireless Commun. Lett., vol. 5, no. 3, pp. 256259, Jun. 2016.

[30] Hewlett-Packard, "Fast switching PIN diodes - Application note 929," Nov. 1999. [Online]. Available: https://www.keysight.com

[31] B. Wang and F. Zhang, "Some inequalities for the eigenvalues of the product of positive semidefinite Hermitian matrices," Linear Algebra Appl., vol. 160, pp. 113-118, 1992.

[32] 3GPP, "TS 38.211: NR - Physical channels and modulation (rel 15)," Sep. 2017. [Online]. Available: http://www.3gpp.org/ftp//Specs/ archive/38_series/38.211/38211-100.zip

[33] 3GPP, "TS 38.214: NR - Physical layer procedures for data (rel. 15)," Sep. 2017. [Online]. Available: http://www.3gpp.org/ftp// Specs/archive/38_series/38.214/38214-f00.zip

[34] Z. Li, D. Rodrigo, L. Jofre, and B. Cetiner, "A new class of antenna array with a reconfigurable element factor," IEEE Trans. Antennas Propag., vol. 61, no. 4, pp. 1947-1955, Apr. 2013.

[35] D. Rodrigo, B. Cetiner, and L. Jofre, "Frequency, radiation pattern and polarization reconfigurable antenna using a parasitic pixel layer," IEEE Trans. Antennas Propag., vol. 62, no. 6, pp. 3422-3427, Jun. 2014.

[36] Z. Li, E. Ahmed, A. Eltawil, and B. Cetiner, "A beam-steering reconfigurable antenna for WLAN applications," IEEE Trans. Antennas Propag., vol. 63, no. 1, pp. 24-32, Jan. 2015.

[37] F. Ferrero, C. Luxey, G. Jacquemod, R. Staraj, and V. Fusco, "A reconfiguralbe hybrid coupler circuit for agile polarisation antenna," in Proc. 1st Eur. Conf. Antennas Propag., Nov. 2006, pp. 1-5.

[38] P. Kysti et al., "IST-4-027756 WINNER II D1.1.2 v.1.1: WINNER II channel models," CELTIC/WINNER Project, Tech. Rep., Sep. 2007.

[39] S. Jaeckel, K. Borner, L. Thiele, and V. Jungnickel, "A geometric polarization rotation model for the 3-D spatial channel model," IEEE Trans. Antennas Propag., vol. 60, no. 12, pp. 5966-5977, Dec. 2012

[40] K. Maliatsos and A. Kanatas, "Modifications of the IST-WINNER channel model for beamspace processing and parasitic arrays," in Proc. 7th Eur Conf. Antennas Propag., Apr. 2013, pp. 989-993. 


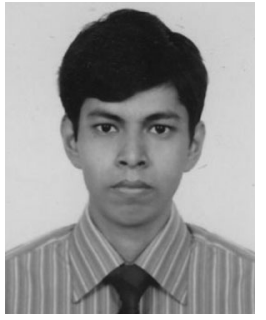

Mehedi Hasan received the B.Sc. degree in electrical engineering from the Bangladesh University of Engineering and Technology, Dhaka, Bangladesh, and the $\mathrm{Ph} . \mathrm{D}$. degree in electrical engineering from Utah State University, Logan, UT, USA, in 2018. From 2013 to 2015, he was with Samsung R\&D Institute Bangladesh. He is currently a Senior System Engineer with Marvell Semiconductor Inc., Santa Clara, CA, USA. His current research interest are in SerDes systems and signal processing for his high speed data communications.

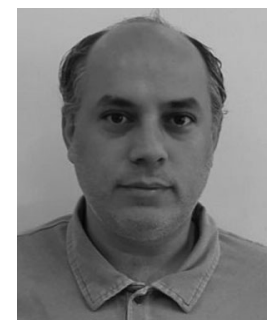

Israfil Bahceci (S'01-M'06) received the B.S. degree in electrical engineering from Bilkent University, Ankara, Turkey, in 1999, the M.S. degree in electrical engineering from Arizona State University, Tempe, AZ, USA, in 2001, and the Ph.D. degree in electrical engineering from the Georgia Institute of Technology, Atlanta, GA, USA, in 2005. He is currently a 5G RAN Developer with Ericsson Canada R\&D, Kanata, ON, Canada. He was a Postdoctoral Fellow with the University of Waterloo, Waterloo, ON, Canada, from 2005 to 2007. He worked as an R\&D Engineer with Nortel Networks, Canada (2007-2009), and Huawei Technologies, Canada (2009-2011), and as an Assistant Professor with the TOBB University of Economics and Technology from 2011 to 2015. From 2015 to 2017, he was a Research Scientist with the Department of Electrical and Computer Engineering, Utah State University, Logan, UT, USA, and he acted as Vice President for i5 Technologies, Inc., Logan, UT, USA. His current research interests include systems, with a particular focus on communication and signal processing, wireless and mobile communications, distributed estimation/detection, and reconfigurable antenna systems for $5 \mathrm{G}$ and beyond systems.

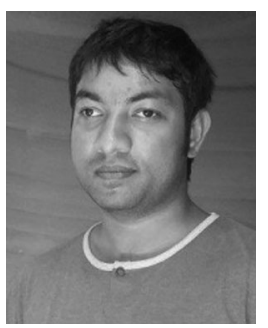

Md. Asaduzzaman Towfiq received the B.S. degree in electrical and electronics engineering from the Bangladesh University of Engineering and Technology, Dhaka, Bangladesh, in 2013, and the Ph.D. degree in electrical engineering from Utah State University, Logan, UT, USA, in 2018. He is currently a Senior Engineer and Vice President with the i5 Technologies, Inc., Logan, UT, USA. His research interests include reconfigurable antenna, phased array, and mmWave antennas.

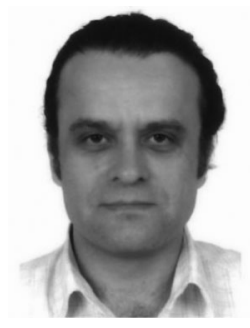

Tolga M. Duman (S'95-M'98-SM'03-F'11) received the B.S. degree from Bilkent University, Ankara, Turkey, in 1993, and the M.S. and Ph.D. degrees from Northeastern University, Boston, MA, USA, in 1995 and 1998, respectively, all in electrical engineering.

He is currently a Professor of Electrical and Electronics Engineering Department, Bilkent University. Prior to joining Bilkent University in September 2012, he was with the School of ECEE at Arizona State University as an Assistant Professor from 1998 to 2004, an Associate Professor from 2004 to 2008, and a Professor after 2008. His current research interests are in systems, with particular focus on communication and signal processing, including wireless and mobile communications, coding/modulation, coding for wireless communications, data storage systems, and underwater acoustic communications.

Dr. Duman was the recipient of the National Science Foundation CAREER Award and the IEEE Third Millennium Medal. He served as an Editor of the IEEE TRANSACTIONS ON WIRELESS COMMUNICATIONS from 2003 to 2008, IEEE COMMUNICATIONS SURVEYS AND TUTORIALS from 2002 to 2007, IEEE TRANSACTIONS ON COMMUNICATIONS from 2007 to 2012, and Physical Communication (Elsevier) from 2010 to 2016. He has been the coding and information theory Area Editor of IEEE TRANSACTIONS ON COMMUNICATIONS since 2011, an Editor of IEEE TRANSACTIONS ON WIRELESS COMMUNICATIONS and the Editor-in-Chief of Physical Communication (Elsevier) since 2016.

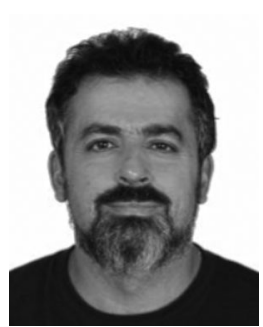

Bedri A. Cetiner (M'99-SM'18) received the Ph.D. degree in electronics and communications engineering from Yildiz Technical University, Istanbul, Turkey, in 1999. He was a North Atlantic Treaty Organization Science Fellow with the University of California at Los Angeles, Los Angeles, CA, USA from 1999 to 2000. From 2000 to 2004, he was a Research Scientist with the Electrical Engineering and Computer Science Department, University of California at Irvine, Irvine, CA, USA. From 2004 to 2007, he was an Assistant Professor with the Department of Space Science and Engineering, Morehead State University, Morehead, KY, USA. In 2007, he joined Utah State University, Logan, UT, USA, where he is currently a Professor with the Department of Electrical Engineering. He is also a Founder, President, and CEO of i5 Technologies Inc., Logan, UT, USA. He is the Principal Inventor of nine patented technologies in the area of wireless communications. His current research interests include the applications of microand nanotechnologies to a new class of microwave/millimeter-wave circuits and systems, and intelligent wireless communications systems with an emphasis on multifunctional reconfigurable antenna equipped MIMO systems. He is a member of the IEEE Antennas and Propagation, Microwave Theory and Techniques, and Communication Societies. 